JOURNAL OF

SYMPLECTIC GEOMETRY

Volume 3, Number 1, 17-54, 2005

\title{
LOCALIZATION FOR THE NORM-SQUARE OF THE MOMENT MAP AND THE TWO-DIMENSIONAL YANG-MILLS INTEGRAL
}

\author{
Chris T. WOODWARD
}

\section{Introduction}

The first seven sections of the paper contain a version of localization for the norm-square of the moment map in equivariant de Rham theory. The main Theorem 5.1 expresses the push-forward of an equivariant cohomology class on a Hamiltonian $K$-manifold with proper moment map as a sum of contributions from fixed point components of one-parameter subgroups corresponding to the critical values of the norm-square of the moment map. If the critical set of the norm-square is non-degenerate in a sense explained below, there is an improved result Theorem 6.1 which expresses the pushforward as an integral over the quotient of the critical set. Many of the ingredients appear in the papers of Paradan $[\mathbf{3 5}, \mathbf{3 6}, \mathbf{3 7}]$, who proved a version of the same result. The proof given here is different from Paradan's. The existence of a localization formula, but not the precise form of the contributions, was first suggested by Witten [47] in his study of two-dimensional Yang-Mills theory. Later Jeffrey and Kirwan [20] gave a formula which had a similar purpose but was expressed in terms of rather different fixed point data. A $K$-theory version was given by Vergne [45] and Paradan [37]. A similar result for sheaf cohomology that I learned from Teleman is explained in the eighth section. This paper arose out of an attempt to bring the theory for de Rham cohomology to the same level. The attempt was not entirely successful, mainly because the formula depends on the existence of pushforwards over non-compact manifolds which are more naturally defined in sheaf cohomology.

The ninth section contains a definition and computation of the YangMills path integral in two dimensions. The idea is to reverse the logic in Witten's [47] paper and take the "stationary phase approximation" (that is, the localization formula) as the definition of the path integral. In order 
to compute it, we apply a symmetry argument from Teleman-Woodward [44] which reduces the computation to an integration over Jacobians. The result is what the physicists call the Migdal formula for the path integral; its large coupling (topological) limit is the Witten volume formula. More general formulas for intersection pairings and indices on the moduli space are given in $[\mathbf{2 1}, \mathbf{3 2}, \mathbf{4 4}]$. A "combinatorial" definition and computation of the two-dimensional Yang-Mills measure, including observables, is given by Levy [27]. Putting these results together shows that the "stationary phase" and "combinatorial" definitions of the two-dimensional Yang-Mills integral are equal. This might be seen as the two-dimensional analog of the much harder conjecture regarding the three-dimensional Chern-Simons path integral, that the "combinatorial" definition via Reshetikhin-Turaev [40] agrees with the "stationary phase" definition of Axelrod-Singer [3] (with leading order term given as in $[\mathbf{1 2}]$ ).

Appendix A contains a proof of an unpublished result of Duistermaat that the gradient flow of minus the norm-square of the moment map converges.

\section{Localization for one-parameter subgroups}

Let $K$ be a compact connected Lie group with Lie algebra $\mathfrak{k}$ and $M$ a $K$-manifold. The equivariant de Rham cohomology $H_{K}(M)$ of $M$ (complex coefficients) can be computed in the Cartan model,

$$
\Omega_{K}(M):=\left(S\left(\mathfrak{k}^{*}\right) \otimes \Omega(M)\right)^{K},
$$

where $\Omega(M)$ denotes the space of smooth forms on $M$ and $S\left(\mathfrak{k}^{*}\right)$ the symmetric algebra on $\mathfrak{k}^{*}$, see $[\mathbf{1 6}]$. The equivariant differential $d_{K}$ can be written

$$
d_{K}: \Omega_{K}(M) \rightarrow \Omega_{K}(M), \quad\left(d_{K} \eta\right)(\zeta)=\left(d+2 \pi i \iota\left(\zeta_{M}\right)\right)(\eta(\zeta)), \quad \zeta \in \mathfrak{k},
$$

where $\zeta_{M} \in \operatorname{Vect}(M)$ denotes the generating vector field $\zeta_{M}(m)=$ $[\exp (-t \zeta) m]$ and $\iota\left(\zeta_{M}\right)$ contraction with $\zeta_{M}$.

If $K$ acts locally freely on $M$, then the equivariant cohomology $H_{K}(M)$ is isomorphic to $H(K \backslash M)$ via pull-back $p^{*}$ by the projection $p: M \rightarrow K \backslash M$. Cartan's homotopy inverse to $p^{*}$ is constructed as follows. Let

$$
\alpha \in \Omega^{1}(M, \mathfrak{k})^{K}, \quad \iota\left(\xi_{M}\right) \alpha=-\xi, \forall \xi \in \mathfrak{k}
$$

be a connection 1-form on $M$ and

$$
\operatorname{curv}(\alpha) \in \Omega^{2}(K \backslash M, M(\mathfrak{k})), \quad p^{*} \operatorname{curv}(\alpha)=d \alpha+\frac{1}{2}[\alpha, \alpha]
$$

denote its curvature. Let

$$
\pi_{\alpha}: \Omega(M) \rightarrow p^{*} \Omega(K \backslash M)
$$

be the horizontal projection defined by $\alpha$. The map

$$
\Omega_{K}(M) \rightarrow \Omega(M)^{K}, \quad \eta \otimes h \mapsto\left(\left(\pi_{\alpha} \eta\right) \otimes h\left(\frac{p^{*} \operatorname{curv}(\alpha)}{2 \pi i}\right)\right)
$$


has image contained in the space of basic forms and descends to a map $\Omega_{K}(M) \rightarrow \Omega(K \backslash M)$ which is a homotopy inverse to $p^{*}$.

Suppose that $M$ is compact and oriented. Integration over $M$ vanishes on equivariant exact forms and defines a push-forward

$$
I_{M, K}: H_{K}(M) \rightarrow S\left(\mathfrak{k}^{*}\right)^{K} .
$$

We also denote by $I_{M, K}(\eta)$ the push-forward of the cohomology class of an equivariant form $\eta$.

For any $K$-equivariant real oriented vector bundle $E$ of even dimension $2 n$, let $\operatorname{Eul}(E) \in H_{K}^{2 n}(M)$ denote its equivariant Euler class, defined as follows. Equip $E$ with a Euclidean metric, let $\mathcal{F}(E)$ denote the orthogonal frame bundle of $E$, and let $\alpha \in \Omega^{1}(\mathcal{F}(E), \mathfrak{s o}(2 n))^{K}$ be a $K$-invariant connection 1-form for $E$. For any $\zeta \in \mathfrak{k}$, the pairing $\alpha\left(\zeta_{\mathcal{F}(E)}\right)$ is $K$-invariant and descends to a map

$$
\phi: M \rightarrow \operatorname{Hom}(\mathfrak{k}, \mathfrak{s o}(E)) .
$$

The form $\operatorname{curv}_{\mathfrak{k}}(E) \in \Omega_{K}^{2}(M, \mathfrak{s o}(E))$ defined by

$$
\operatorname{curv}_{\mathfrak{k}}(E):=\operatorname{curv}(E)+2 \pi i \phi
$$

is the equivariant curvature of $E$. The Euler class of $E$ is

$$
\operatorname{Eul}(E):=\operatorname{Pf}\left(\frac{\operatorname{curv}_{\mathfrak{k}}(E)}{2 \pi}\right) \in \Omega_{K}^{2 n}(M),
$$

where Pf is the Pfaffian and the right-hand side denotes the Chern-Weil characteristic form.

Let $K$ be a torus. If $E$ is a complex $K$-representation, then $E$ splits into a sum of weight spaces $E_{\mu}$ for $\mu \in \mathfrak{k}^{*}$, so that $\exp (\xi) v=e^{2 \pi i \mu(\xi)} v$ for $v \in E_{\mu}$ and $\xi \in \mathfrak{k}$. If $E$ is a real even-dimensional representation of $K$, then $E$ admits an invariant complex structure and the weights $\mu_{1}, \ldots, \mu_{n}$ are independent of the choice of complex structure up to sign. If $E$ is oriented, then the product of the complex weights is determined by the orientation on $E$ and

$$
(\operatorname{Eul}(E))(\xi)=\prod_{j=1}^{n}-2 \pi i \mu_{j}(\xi) .
$$

We will also need cohomology with smooth and distributional coefficients. An equivariant form with smooth coefficients is a smooth equivariant map $\mathfrak{k} \rightarrow \Omega(M)$. The equivariant differential extends to equivariant forms with smooth coefficients and its cohomology is the equivariant cohomology of $M$ with smooth coefficients. Let $C_{0}^{\infty}\left(\mathfrak{k}^{*}\right)$ denote the space of compactly supported smooth functions on $\mathfrak{k}^{*}$, and $D^{\prime}\left(\mathfrak{k}^{*}\right)$ the space of distributions on $\mathfrak{k}^{*}$, that is, the space of continuous linear forms on $C_{0}^{\infty}\left(\mathfrak{k}^{*}\right)$. Let $\mathcal{S}\left(\mathfrak{k}^{*}\right)$ denote the space of Schwartz functions on $\mathfrak{k}^{*}$, the space of smooth functions $f$ such that for any polynomial differential operator $P$, the function $\mathrm{Pf}$ is bounded. Its dual $\mathcal{S}^{\prime}\left(\mathfrak{k}^{*}\right)$ is the space of tempered distributions on $\mathfrak{k}^{*}$. The inclusion 
$C_{0}^{\infty}\left(\mathfrak{k}^{*}\right) \subset \mathcal{S}\left(\mathfrak{k}^{*}\right)$ dualizes to an injection $S^{\prime}\left(\mathfrak{k}^{*}\right) \rightarrow \mathcal{D}^{\prime}\left(\mathfrak{k}^{*}\right)$. The symmetric algebra $S\left(\mathfrak{k}^{*}\right)$ embeds in $\mathcal{S}^{\prime}\left(\mathfrak{k}^{*}\right)$ via Fourier transform as the space of distributions supported at the identity. An equivariant differential form with distributional coefficients is an equivariant continuous linear map from $C_{0}^{\infty}\left(\mathfrak{k}^{*}\right)$ to $\Omega(M)$. Let $\mathcal{C}_{K}(M)$ denote the complex of such forms; the equivariant differential extends to $\mathcal{C}_{K}(M)$ and its cohomology $\mathcal{H}_{K}(M)$ is the equivariant cohomology with distributional coefficients. The basic results on equivariant cohomology with distributional coefficients are discussed in detail in Kumar-Vergne [24]. If $M$ is compact and oriented, then the map $I_{M, K}$ extends to $I_{M, K}: \mathcal{H}_{K}(M) \rightarrow \mathcal{D}^{\prime}\left(\mathfrak{k}^{*}\right)^{K}$.

In order to state the localization formula, we need to discuss inversion of the Euler class. Suppose that $E$ is an oriented real vector bundle of even dimension and that a circle subgroup $U(1)_{\zeta} \subset G$ generated by $\zeta \in \mathfrak{k}$ acts trivially on $M$ fixing only the zero section in $E$. According to Atiyah-Bott [1], the Euler class is invertible after suitably modifying the coefficient ring. For distributional coefficients, the construction is carried out in Paradan [35, Section 4]. A definition equivalent to Paradan's goes as follows. Since $\zeta$ acts with non-zero weights, $\operatorname{Pf}(\phi(m, \xi))$ is a hyperbolic polynomial, that is,

$$
\operatorname{Pf}(\phi(m, \xi+i \tau \zeta)) \neq 0, \quad \forall \xi \in \mathfrak{k}, \tau<0, m \in M .
$$

By a standard result in distribution theory [19, Theorem 12.5.1], $\operatorname{Pf}(\phi(m, \cdot))^{-k}$ has a unique distributional extension with support on $(\zeta, \cdot) \geq$ 0 for any $k>0$. Let $\operatorname{Pf}\left(\operatorname{curv}_{\mathfrak{k}}(E) / 2 \pi\right)_{+}$denote the terms containing forms on $M$ of positive degree, so that

$$
\operatorname{Pf}\left(\frac{\operatorname{curv}_{\mathfrak{k}}(E)}{2 \pi}\right)=\operatorname{Pf}(i \phi)+\operatorname{Pf}\left(\frac{\operatorname{curv}_{\mathfrak{k}}(E)}{2 \pi}\right)_{+} .
$$

Define

$$
\operatorname{Eul}(E)_{\zeta}^{-1}:=\operatorname{Pf}(i \phi)^{-1}\left(1+\frac{\operatorname{Pf}\left(\operatorname{curv}_{\mathfrak{k}}(E) / 2 \pi\right)_{+}}{\operatorname{Pf}(i \phi)}\right)^{-1}
$$

interpreted via its power series expansion, which is finite since $\operatorname{Pf}\left(\operatorname{curv}_{\mathfrak{k}}(E)\right)_{+}$ is nilpotent.

For any $\zeta \in \mathfrak{k}$, let $M^{\zeta}$ denote the fixed point set of the one-parameter subgroup $U(1)_{\zeta}$ generated by $\zeta$. Fix orientations on $M^{\zeta}$ and $T_{M \zeta} M$ which induce the given orientation on $T M \mid M^{\zeta}$. If $E$ is a $K$-equivariant vector bundle and $K^{\prime} \subset K$ is a subgroup stabilizing a submanifold $M^{\prime} \subset M$, then $\operatorname{Res}_{M^{\prime}, K^{\prime}}^{M, K} E$ denotes the restriction of $E$ to a $K^{\prime}$-equivariant bundle on $M^{\prime}$. Similarly, if $\eta$ is a $K$-equivariant cohomology form or class, we denote by $\operatorname{Res}_{M^{\prime}, K^{\prime}}^{M, K} \eta$ the restriction of $\eta$ to a $K^{\prime}$-equivariant form or class on $M^{\prime}$.

Theorem 2.1. (Localization for one-parameter subgroups) For any compact oriented $K$-manifold $M, \eta$ an equivariant cohomology class with smooth 
coefficients, and $\zeta \in \mathfrak{k}$,

$$
I_{M, K_{\zeta}}\left(\operatorname{Res}_{K_{\zeta}}^{K} \eta\right)=I_{M^{\zeta}, K_{\zeta}}\left(\operatorname{Res}_{M^{\zeta}, K_{\zeta}}^{M, K} \eta \wedge \operatorname{Eul}\left(T_{M^{\zeta}} M\right)_{\zeta}^{-1}\right) .
$$

For smooth values of $\operatorname{Eul}_{\zeta}^{-1}\left(T_{M \zeta} M\right)$, Theorem 2.1 is proved in AtiyahBott [2] and Berline-Vergne [5]. For the distributional version, see Guillemin-Lerman-Sternberg [13], Canas-Guillemin [8], and Paradan [35, Section 5].

\section{Hamiltonian $K$-manifolds}

Let $T \subset K$ be a maximal torus, $\mathfrak{t}$ its Lie algebra, and $\mathfrak{t}^{*}$ its dual. Let $\mathfrak{t}^{*} \rightarrow \mathfrak{k}^{*}$ be the injection whose image is the fixed point set of $T$. Choose a closed positive Weyl chamber $\mathfrak{t}_{+}$. Using an invariant metric on $\mathfrak{k}$ to identify $\mathfrak{t}^{*}$ with $\mathfrak{t}$, let $\mathfrak{t}_{+}^{*}$ denote the image of $\mathfrak{t}_{+}$in $\mathfrak{t}^{*}$; this is independent of the choice of metric. If $N$ is a right $K$-manifold, then by $N \times_{K} M$ we mean the quotient of $N \times M$ by the $K$-action $k(n, m)=\left(n k^{-1}, k m\right)$.

3.1. Basic definitions and results. A Hamiltonian $K$-manifold consists of a smooth $K$-manifold $M$, a symplectic form $\omega$, and an equivariant moment $\operatorname{map} \Phi: M \rightarrow \mathfrak{k}^{*}$ satisfying

$$
\iota\left(\xi_{M}\right) \omega=-d(\Phi, \xi), \quad \forall \xi \in \mathfrak{k} .
$$

If $\omega$ is closed but degenerate the data are called a degenerate Hamiltonian $K$-manifold. We denote by $K_{M} \subset K$ the principal stabilizer, that is, the stabilizer of a generic element in $\Phi^{-1}\left(\mathfrak{t}_{+}^{*}\right)$. The principal orbit-type stratum for $K$ resp. $\mathfrak{k}$ is the set of points $m \in M$ with $K_{m}$ conjugate to $K_{M}$ resp. $\mathfrak{k}_{m}$ conjugate to $\mathfrak{k}_{M}$. For references on the following, see $[\mathbf{2 3}, \mathbf{2 6}]$.

Theorem 3.1. Let $M$ be a connected Hamiltonian $K$-manifold with proper moment map.

a) (Kirwan convexity) The intersection $\Delta(M):=\Phi(M) \cap \mathfrak{t}_{+}^{*}$ is a convex polyhedron called the moment polyhedron of $M$.

b) (Principal cross-section) The unique minimal open face $\sigma(M)$ of $\mathfrak{t}_{+}^{*}$ containing $\Delta(M)$ in its closure is the principal face for $M$. The inverse image $\Phi^{-1}(K \sigma(M))$ is an open subset of $M$ whose complement is codimension at least two, and the $\operatorname{map} K \times_{K_{\sigma}} \Phi^{-1}(\sigma(M)) \rightarrow M,[k, m] \mapsto$ $\mathrm{km}$ is a diffeomorphism onto its image.

The rank of $M$ is the dimension of $\Delta(M) . M$ is maximal rank if and only if $\mathfrak{k}_{M}$ is trivial.

The moment map condition (3.1) is equivalent to the condition that the equivariant symplectic form

$$
\omega_{K}(\xi)=\omega+2 \pi i(\Phi, \xi) \in \Omega_{K}(M)
$$


is equivariantly closed. The equivariant Liouville form is

$$
\mathcal{L}:=\exp \left(\omega_{K}\right)=\exp (\omega) \exp (2 \pi i(\Phi, \xi)) .
$$

Let $M$ be a Hamiltonian $K$-manifold with proper moment map. The Duistermaat-Heckman measure $\mu_{M, K}$ is the push-forward of the measure defined by the top degree component of $\exp (\omega)$ under $\Phi$,

$$
\mu_{M, K}:=\Phi_{*}(\exp (\omega)) \in \mathcal{D}^{\prime}\left(\mathfrak{k}^{*}\right)^{K} .
$$

More generally, suppose that $\eta \in \Omega_{K}(M)$ is closed. If $M$ is compact, we define the twisted Duistermaat-Heckman distribution as the Fourier transform of the push-forward of $\mathcal{L} \wedge \eta$ :

$$
\mu_{M, K}(\eta)=\mathcal{F}_{\mathfrak{k}}\left(I_{M, K}(\mathcal{L} \wedge \eta)\right) .
$$

If $M$ is not necessarily compact, suppose that $\xi_{1}, \ldots, \xi_{\operatorname{dim}(\mathfrak{k})}$ are coordinates on $\mathfrak{k}$ and that $\eta=\sum_{I} \eta_{I} \xi_{I}$, where $I$ ranges over multisets with elements $1, \ldots, \operatorname{dim}(\mathfrak{k})$ and $\xi_{I}:=\prod_{i \in I} \xi_{i}$. Define

$$
\mu_{M, K}(\eta):=\sum_{I} \partial_{I} \Phi_{*}\left(\exp (\omega) \wedge \eta_{I}\right) \in \mathcal{D}^{\prime}\left(\mathfrak{k}^{*}\right)^{K},
$$

where $\partial_{I}=\mathcal{F}_{\mathfrak{k}}\left(\xi_{I}\right)$, the Fourier transform of $\xi_{I} \cdot \mu_{M, K}(\eta)$ is supported on the image of $\Phi$ and depends only on the cohomology classes of $\omega_{K}$ and $\eta$.

Later we will need a variation of this construction when $M$ is a compact Hamiltonian $K$-manifold with boundary. Let $\eta \in \Omega_{K}(M)$ be closed and $\alpha \in$ $\Omega_{K}^{1}(M)=\Omega^{1}(M)^{K}$ an invariant 1-form. Consider the family of (possibly degenerate) symplectic forms and moment maps

$$
\omega_{s}=\omega+s d \alpha, \quad\left(\Phi_{s}(m), \xi\right)=(\Phi(m), \xi)+s \alpha\left(\xi_{M}\right) .
$$

Let $\mu_{M, K, s}(\eta)$ denote the corresponding twisted Duistermaat-Heckman distribution.

Proposition 3.2. Let $h \in C_{0}^{\infty}\left(\mathfrak{k}^{*}\right)^{K}$ be such that $\operatorname{supp}(h) \cap \Phi_{s}(\partial M)$ is empty for $s \in[0,1] .\left(\mu_{M, K, s}(\eta), h\right)$ is independent of $s \in[0,1]$.

This follows from the same argument as in the case without boundary, since the relevant integrands are supported on the interior.

3.2. Coadjoint orbits. The following material is mostly covered in Berline-Getzler-Vergne [4, Section 7.5]. We parametrize coadjoint orbits by their intersection with the positive chamber:

$$
\mathfrak{t}_{+}^{*} \rightarrow K \backslash \mathfrak{k}^{*}, \quad \lambda \mapsto K \lambda .
$$

A symplectic form on $K \lambda$ is defined by the Kirillov-Kostant-Souriau formula

$$
\omega_{m}\left(\xi_{M}(m), \zeta_{M}(m)\right)=(m,[\xi, \zeta]) .
$$


The action of $K$ on $K \lambda$ is Hamiltonian with moment map given by the inclusion into $\mathfrak{k}^{*}$. The weights on the tangent space at a $T$-fixed point $w \lambda$ are the roots $\alpha$ with $(\alpha, w \lambda)>0$. By localization (2.1)

$$
\left(I_{K \cdot \lambda, T}(\mathcal{L})\right)(\zeta)=\sum_{[w] \in W / W_{\sigma}} \frac{\exp (2 \pi i(w \lambda, \zeta))}{\prod_{(\alpha, w \lambda)<0} 2 \pi i(\alpha, \zeta)}
$$

Let $\rho$ denote the half-sum of positive roots of $\mathfrak{k}$. The symplectic volume of $K \cdot \lambda$ is the equal to

$$
\begin{aligned}
\operatorname{Vol}(K \cdot \lambda) & =\left(I_{K \cdot \lambda, T}(\mathcal{L})\right)(0) \\
& =\lim _{t \rightarrow 0}\left(I_{K \cdot \lambda, T}(\mathcal{L})\right)(t \rho) \\
& =\frac{\prod_{(\alpha, \lambda)<0}(\alpha, \lambda)}{\prod_{(\alpha, \lambda)<0}(\alpha, \rho)} .
\end{aligned}
$$

A computation at the tangent space at $\lambda$ shows that the symplectic and Riemannian volumes are related by

$$
\operatorname{Vol}(K \cdot \lambda)=(2 \pi)^{\operatorname{dim}\left(K / K_{\lambda}\right) / 2} \prod_{(\alpha, \lambda)>0}(\alpha, \lambda) \operatorname{Vol}\left(K / K_{\lambda}\right),
$$

which implies

$$
\operatorname{Vol}\left(K / K_{\lambda}\right)^{-1}=(2 \pi)^{\operatorname{dim}\left(K / K_{\lambda}\right) / 2} \prod_{(\alpha, \lambda)>0}(\alpha, \rho) .
$$

Similarly, let $\operatorname{RVol}(K \cdot \lambda)$ denote the volume of $K \cdot \lambda$ with respect to the Riemannian metric induced by the embedding $K \cdot \lambda \rightarrow \mathfrak{k}^{*}$. We have

$$
\operatorname{RVol}(K \cdot \lambda)=(2 \pi)^{\operatorname{dim}\left(K / K_{\lambda}\right)} \prod_{(\alpha, \lambda)>0}(\alpha, \lambda)^{2} \operatorname{Vol}\left(K / K_{\lambda}\right) .
$$

If $K_{\lambda}=T$, then $\operatorname{RVol}(K \cdot \lambda)=\Pi(\lambda)^{2} \operatorname{Vol}\left(K / K_{\lambda}\right)$, where

$$
\Pi(\xi)=\prod_{\alpha>0} 2 \pi(\alpha, \xi)=i^{-\operatorname{dim}(K / T) / 2} \operatorname{Eul}(\mathfrak{k} / \mathfrak{t}) .
$$

3.3. Symplectic quotients. The symplectic quotient of $M$ at $\lambda \in \mathfrak{k}^{*}$ is

$$
M_{(\lambda)}:=K_{\lambda} \backslash \Phi^{-1}(\lambda) .
$$

If $\Phi^{-1}(\lambda)$ is contained in the principal orbit-type stratum for $K$ (resp. $\mathfrak{k}$ ), then $M_{(\lambda)}$ has the structure of a symplectic manifold (resp. orbifold), with symplectic form $\omega_{(\lambda)}$ the unique 2 -form that pulls back to the restriction of $\omega$ to $\Phi^{-1}(\lambda)$. For $\lambda$ such that $\Phi^{-1}(\lambda)$ is contained in the principal orbit-type stratum, we denote by $\mathcal{L}_{(\lambda)}=\exp \left(\omega_{(\lambda)}\right)$ the Liouville form on $M_{(\lambda)}$.

The relation between the cohomology of $M$ and the cohomology of the quotient was studied by Kirwan [22]. Let $\lambda$ be a regular value of $\Phi$ and 
$\kappa_{\lambda}$ the composition of restriction $H_{K}(M) \rightarrow H_{K}\left(\Phi^{-1}(\lambda)\right)$ with the isomorphism $H_{K}\left(\Phi^{-1}(\lambda)\right) \rightarrow H\left(M_{(\lambda)}\right)$. $\kappa_{\lambda}$ extends to cohomology with smooth coefficients. By $[\mathbf{2 2}]$, if $\lambda$ is central and $M$ is compact, then $\kappa_{\lambda}$ is surjective.

The cohomological pairings on the symplectic quotient are encoded in the twisted Duistermaat-Heckman distributions. Let $\mu_{\mathfrak{k}^{*}}$ and $\mu_{\mathfrak{t}^{*}}$ denote the Lebesgue measures induced by the metrics on $\mathfrak{k}^{*}$ and $\mathfrak{t}^{*}$, respectively.

Proposition 3.3. Let $M$ be a (possibly degenerate) Hamiltonian $K$-manifold with proper moment map $\Phi$. Let $\eta \in \Omega_{K}(M)$ be a closed equivariant form. Let $U \subset \mathfrak{k}^{*}$ be a subset such that $K$ acts locally freely on $\Phi^{-1}(U)$, and $U^{\text {reg }} \subset U$ the set of regular values of $\Phi$ in $U$.

a) $\left.\mu_{M, K}(\eta)\right|_{U}=\left.\operatorname{Vol}\left(K / K_{M}\right) \operatorname{Vol}(K \cdot \lambda)^{-1} I_{M_{(\lambda)}}\left(\mathcal{L}_{(\lambda)} \wedge \kappa_{\lambda}(\eta)\right) \mu_{\mathfrak{k}^{*}}\right|_{U}$.

b) $I_{M_{(\lambda)}}\left(\mathcal{L}_{(\lambda)} \wedge \kappa_{\lambda}(\eta)\right)$ is equal to a polynomial in $\lambda$ on any subset of $U^{\text {reg }}$ on which $K_{\lambda}$ is constant.

c) Let $\lambda \in \mathfrak{t}_{+}^{*}$ be a regular value of $\Phi$. For $\nu \in \mathfrak{t}_{\mathrm{reg}}^{*} \cap \mathfrak{t}_{+}^{*}, \lim _{\nu \rightarrow \lambda}\left(\# W_{\lambda}\right)^{-1}$ $\mathcal{I}_{M_{(\nu)}}\left(\mathcal{L}_{(\nu)} \wedge \kappa_{\nu}\left(\eta \wedge \operatorname{Eul}\left(\left(\mathfrak{k}_{\lambda} / \mathfrak{t}\right)^{*}\right)\right)\right)=I_{M_{(\lambda)}}\left(\mathcal{L}_{(\lambda)} \wedge \kappa_{\lambda}(\eta)\right)$.

Proof. In the non-degenerate case, (a) and (b) are basic results of Duistermaat-Heckman, see $[\mathbf{1 1}, \mathbf{2 0}]$. The degenerate cases are similar, using the machinery of equivariant cohomology instead of local models, as in Atiyah-Bott [2]: if $\lambda_{1}, \lambda_{2} \in U^{\text {reg }}$ and $K_{\lambda_{1}}=K_{\lambda_{2}}$, then let $A \subset \mathfrak{k}^{*}$ be a one-manifold (arc) with boundary $\partial A=\left\{\lambda_{1}, \lambda_{2}\right\}$, such that $A$ is contained in $U$ and $K_{\lambda}=K_{\lambda_{1}}$ for all $\lambda \in A$. Suppose that the inverse image $\Phi^{-1}(A)$ is smooth. Since $K$ acts locally freely, the quotient

$$
M_{A}=K_{\lambda_{1}} \backslash \Phi^{-1}(A)
$$

is a smooth orbifold. By equation (2.1), the equivariant symplectic form $\tilde{\omega}$ as well as $\eta$ descend to closed forms on $M_{A}$. We have

$$
\mathcal{L}_{\lambda_{i}}=\kappa_{\lambda_{1}}\left(\exp \left(\tilde{\omega}-2 \pi i\left(\lambda_{i}, \xi\right)\right)\right), \quad i=1,2,
$$

and therefore, by Stokes theorem applied to $M_{A}$

$$
I_{M_{\left(\lambda_{2}\right)}}\left(\mathcal{L}_{\lambda_{2}} \wedge \kappa_{\lambda_{2}}(\eta)\right)=I_{M_{\left(\lambda_{1}\right)}}\left(\mathcal{L}_{\lambda_{1}} \wedge \kappa_{\lambda_{2}}\left(\eta \wedge \exp \left(2 \pi i\left(\lambda_{1}-\lambda_{2}, \xi\right)\right)\right)\right.
$$

which is polynomial in $\lambda_{2}$. Since $\Phi^{-1}(A)$ is smooth for generic $A$, this shows (b). (c) $M_{(\nu)}$ is a $K_{\lambda} / T$-fiber bundle over $M_{(\lambda)}$. The class $\kappa_{\lambda}\left(\operatorname{Eul}\left(\left(\mathfrak{k}_{\lambda} / \mathfrak{t}\right)^{*}\right)\right)$ restricts to the Euler class of the tangent bundle on the fiber $K_{\lambda} / T$, which has Euler characteristic $\#\left(K_{\lambda} / T\right)^{\mathrm{T}}=\# W_{\lambda}$. The result follows from fiber integration.

We will need a more general "reduction in stages" version of this result. Let $K_{1} \subset K$ be a normal subgroup, and $K_{2}=K / K_{1}$ the quotient. Let $\Phi=\left(\Phi_{1}, \Phi_{2}\right)$ be the decomposition of $\Phi$ according to an invariant splitting $\mathfrak{k} \cong \mathfrak{k}_{1} \oplus \mathfrak{k}_{2}$. 
Proposition 3.4. Let $M$ be a (possibly degenerate) Hamiltonian $K$-manifold with proper moment map $\Phi$. Let $\eta \in \Omega_{K}(M)$ be closed and $U_{1} \subset \mathfrak{k}_{1}^{*}, U_{2} \subset \mathfrak{k}_{2}^{*}$ open subsets such that $K_{1}$ acts locally freely on $\Phi^{-1}\left(U_{1} \times U_{2}\right)$. Then

$$
\left.\mu_{M, K}(\eta)\right|_{U_{1} \times U_{2}}=\frac{\operatorname{Vol}\left(K_{1} / K_{1, M}\right)}{\operatorname{Vol}\left(K_{1} \cdot \lambda_{1}\right)} \int_{U_{1}^{\text {reg }}}\left(\left.\mu_{M_{\left(\lambda_{1}\right)}, K_{2}}\left(\kappa_{\lambda_{1}}(\eta)\right)\right|_{U_{2}} \otimes \delta_{\lambda_{1}}\right) d \lambda_{1},
$$

where the integral is over the set $U_{1}^{\mathrm{reg}}$ of regular values of $\Phi_{1}$ in $U_{1}$.

Here, the twisted Duistermaat-Heckman distribution $\mu_{M_{\left(\lambda_{1}\right)}, K_{2}}\left(\eta_{\left(\lambda_{1}\right)}\right)$ on $M_{\left(\lambda_{1}\right)}$ is a distribution on $\mathfrak{k}_{2}^{*}$. Its tensor product with $\delta_{\left(\lambda_{1}\right)}$ is a distribution on $\mathfrak{k}^{*}$ depending on $\lambda_{1}$.

Lemma 3.5. Suppose that in the setting of Proposition $3.4, \mathfrak{k}_{m} \subset \mathfrak{k}_{2}$ for all $m \in \Phi^{-1}\left(U_{1} \times U_{2}\right)$. Then $\left.\mu_{M_{\left(\lambda_{1}\right)}, K_{2}}\left(\kappa_{\lambda_{1}}(\eta)\right)\right|_{U_{2}}$ depends polynomially on $\lambda_{1}$.

Proof. The assumption $\mathfrak{k}_{m} \subset \mathfrak{k}_{2}$ implies that the $K_{1, \lambda_{1}}$-bundle $\Phi_{1}^{-1}\left(\lambda_{1}\right) \rightarrow$ $M_{\left(\lambda_{1}\right)}$ admits a connection 1-form $\alpha$ that vanishes on the generating vector fields for $K_{2}$. (Construct the connection locally, then patch together.) Hence, the $K_{2}$-equivariant form $\operatorname{curv}_{K_{2}} \alpha$ is nilpotent, so $\kappa_{\lambda_{1}}\left(\exp \left(2 \pi i\left(\lambda_{1}, \xi\right)\right)\right)=\exp \left(\left(\lambda_{1}, \operatorname{curv}_{K_{2}} \alpha\right)\right)$ is a polynomial in $\lambda_{1}$. Using the $K_{2}$-equivariant analog of (3.11) implies the result.

We apply this to prove a polynomiality result for non-regular values. Let $\lambda_{0}, \lambda_{1} \in \mathfrak{k}^{*}$ be central and

$$
R_{\lambda_{0}, \lambda_{1}}=\lambda_{0}+\mathbb{R}_{\geq 0}\left(\lambda_{1}-\lambda_{0}\right)
$$

the ray starting from $\lambda_{0}$ to $\lambda_{1}$. Let $\mathfrak{k}_{1}$ denote the span of $\lambda_{1}$, and $\mathfrak{k}_{2}$ the quotient $\mathfrak{k} / \mathfrak{k}_{1}$. We say that a distribution $\mu \in \mathcal{D}^{\prime}\left(\mathfrak{k}^{*}\right)$ is smooth, resp. polynomial along the ray $R_{\lambda_{0}, \lambda_{1}}$ at $\lambda_{2} \in R_{\lambda_{0}, \lambda_{1}}-\left\{\lambda_{0}\right\}$ if $\mu$ is equal to

$$
\int_{\mathfrak{k}_{1}^{*}}\left(\mu_{2}\left(\lambda_{1}\right) \otimes \delta\left(\lambda_{1}\right)\right) d \lambda_{1}
$$

for some distribution $\mu_{2}\left(\lambda_{1}\right) \in \mathcal{D}^{\prime}\left(\mathfrak{k}_{2}^{*}\right)$ depending smoothly, resp. polynomially on $\lambda_{1} \in \mathfrak{k}_{1}^{*}$. We say $\mu$ is smooth, resp. polynomial near $\lambda_{2} \in R_{\lambda_{0}, \lambda_{1}}$ if this holds in a neighborhood of $\lambda_{2}$.

Proposition 3.6. Let $M$ be a (possibly degenerate) Hamiltonian $K$-manifold with proper moment map $\Phi$. Let $\eta \in \Omega_{K}(M)$ be closed. Let $\lambda_{0}, \lambda_{1} \in \mathfrak{k}^{*}$ be distinct and central. Then, $\mu_{M, K}(\eta)$ is polynomial along $R_{\lambda_{0}, \lambda_{1}}$ near $\lambda_{1}$ sufficiently close to $\lambda_{0}$.

Proof. Let $m \in \Phi^{-1}\left(\lambda_{1}\right), \xi \in \mathfrak{k}_{m}$, and $M^{\xi}$ the infinitesimal fixed point set of $\xi$ containing $m$. For $\lambda_{1}$ sufficiently close to $\lambda_{0}, M^{\xi}$ meets $\Phi^{-1}\left(\lambda_{0}\right)$ and so

$$
\left(\lambda_{1}, \xi\right)=\left(\Phi\left(M^{\xi}\right), \xi\right)=\left(\lambda_{0}, \xi\right) .
$$

It follows that $\lambda_{1}-\lambda_{0}$ annihilates $\xi$, hence $\xi$ is contained in $\mathfrak{k}_{2}$. The result now follows from Proposition 3.4 and Lemma 3.5. 
3.4. Induction. First, we define induction for distributions. Let $\tau$ be any face of $\mathfrak{t}_{+}^{*}$. Let $\operatorname{Vol}_{K_{\tau}}^{K}: \mathfrak{t}^{*} \rightarrow \mathbb{R}$ denote the function

$$
\operatorname{Vol}_{K_{\tau}}^{K}(\lambda):=\frac{\operatorname{Vol}(K \cdot \lambda)}{\operatorname{Vol}\left(K_{\tau} \cdot \lambda\right)}, \lambda \in \mathfrak{t}_{+}^{*} .
$$

Using equation (3.7), $\operatorname{Vol}_{K_{\tau}}^{K}$ has a polynomial extension to $\mathfrak{t}^{*}$ that is invariant under $W_{\tau}$. We denote by the same name its extension to $\mathfrak{k}_{\tau}^{*}$. Define

$$
\operatorname{Ind}_{K_{\tau}}^{K}: \mathcal{D}^{\prime}\left(\mathfrak{k}_{\tau}^{*}\right) \rightarrow \mathcal{D}^{\prime}\left(\mathfrak{k}^{*}\right)^{K},\left(\operatorname{Ind}_{K_{\tau}}^{K} \mu, h\right)=\left(\mu, \operatorname{Vol}_{K_{\tau}}^{K} \operatorname{Res}_{K_{\tau}}^{K} h\right) .
$$

Restriction to tempered distributions defines a map $\mathcal{S}^{\prime}\left(\mathfrak{k}_{\tau}^{*}\right) \rightarrow \mathcal{S}^{\prime}\left(\mathfrak{k}^{*}\right)^{K}$. The same notation will be used for the Fourier transform $\mathcal{S}^{\prime}\left(\mathfrak{k}_{\tau}\right) \rightarrow \mathcal{S}^{\prime}(\mathfrak{k})^{K}$. The reader may note that $\operatorname{Ind}_{K_{\tau}}^{K}$ is the "semiclassical limit" of holomorphic induction of representation rings $R\left(K_{\tau}\right) \rightarrow R(K)$.

Next, we define induction for Hamiltonian actions. If $M$ is a Hamiltonian $K_{\tau}$-manifold, one can define a possibly degenerate Hamiltonian $K$-manifold by

$$
\operatorname{Ind}_{K_{\tau}}^{K} M:=K \times_{K_{\tau}} M
$$

with the unique closed equivariant 2 -form $\operatorname{Ind}_{K_{\tau}}^{K} \omega_{K}$ restricting to $\omega_{K}$ on $M$. The 2 -form $\operatorname{Ind}_{K_{\tau}}^{K} \omega$ is degenerate if and only if $\Delta(M)$ lies in the union of open faces of $\boldsymbol{t}_{+}^{*}$ whose closure contains $\tau$.

Finally, we define induction for equivariant forms. The inclusion

$$
M \rightarrow \operatorname{Ind}_{K_{\tau}}^{K} M, \quad m \mapsto[1, m]
$$

induces a map $\Omega_{K}\left(\operatorname{Ind}_{K_{\tau}}^{K} M\right) \rightarrow \Omega_{K_{\tau}}(M)$. A homotopy inverse is provided by the composition $\operatorname{Ind}_{K_{\tau}}^{K}$ of the maps

$$
\Omega_{K_{\tau}}(M) \rightarrow \Omega_{K \times K_{\tau}}(K \times M) \rightarrow \Omega_{K}\left(\operatorname{Ind}_{K_{\tau}}^{K} M\right),
$$

where the last map is the Cartan map (2.1).

The following proposition shows that taking Duistermaat-Heckman distributions commutes with induction, see Paradan [36, 3.13]. For completeness, we include a proof.

Proposition 3.7. Let $\tau$ be a face of the positive Weyl chamber, $M a$ Hamiltonian $K_{\tau}$-manifold with proper moment map, and $\eta$ a closed polynomial $K_{\tau}$-equivariant form on $M . \mu_{\operatorname{Ind}_{K_{\tau}}^{K} M, K}\left(\operatorname{Ind}_{K_{\tau}}^{K} \eta\right)=\operatorname{Ind}_{K_{\tau}}^{K} \mu_{M, K_{\tau}}(\eta)$.

Proof. If the closure of $\tau$ contains the principal face $\sigma$ of $M$, then $\operatorname{Ind}_{K_{\sigma}}^{K}=$ $\operatorname{Ind}_{K_{\tau}}^{K} \operatorname{Ind}_{K_{\sigma}}^{K_{\tau}}$. Therefore, it suffices to prove the proposition for $\tau=\sigma$. Let $\operatorname{conn}_{K_{\sigma}}^{K} \in \Omega^{1}\left(K, \mathfrak{k}_{\sigma}\right)$ be the connection on $K \rightarrow K / K_{\sigma}$ defined using the metric on $\mathfrak{k}, \widetilde{\operatorname{curv}}_{K_{\sigma}}^{K} \in \Omega_{K}^{2}\left(K / K_{\sigma}, K\left(\mathfrak{k}_{\sigma}\right)\right)$ its equivariant curvature and $\operatorname{curv}_{K_{\sigma}}^{K} \in \Omega^{2}\left(K / K_{\sigma}\left(\mathfrak{k}_{\sigma}\right)\right.$ its ordinary curvature. For each $\lambda \in \sigma$, the pairing 
with the curvature defines an equivariant 2 -form $\left(\widetilde{\operatorname{curv}}_{K_{\sigma}}^{K}, \lambda\right) \in \Omega_{K}^{2}\left(K / K_{\sigma}\right)$. Let

$$
p_{i} \in S\left(\mathfrak{k}_{\sigma}\right)^{K_{\sigma}}, \quad i=1,2, \ldots
$$

be a basis for the invariant polynomials on $\mathfrak{k}_{\sigma}$. For each $i$, we have a characteristic form defined via the Chern-Weil homomorphism

$$
\widetilde{\operatorname{curv}_{K_{\sigma}, i}^{K}} \sum_{I} \operatorname{curv}_{K_{\sigma}, i, I}^{K} \xi_{I} \in \Omega_{K}\left(\frac{K}{K_{\sigma}}\right) \text {. }
$$

Because $\left(\operatorname{curv}_{K_{\sigma}}^{K}, \lambda\right)$ is the pull-back of the Kirillov-Kostant-Souriau form (3.3) under the map $K / K_{\sigma} \rightarrow K \cdot \lambda$, we have for any $h \in \mathcal{S}\left(\mathfrak{k}^{*}\right)^{K}$

$$
\sum_{I} \int_{K / K_{\sigma}} \operatorname{curv}_{K_{\sigma}, i, I}^{K} \wedge \exp \left(\operatorname{curv}_{K_{\sigma}}^{K}, \lambda\right)\left(\partial_{I} h\right)(\lambda)=\left(\partial_{i} \operatorname{Vol}_{K_{\sigma}}^{K} h\right)(\lambda),
$$

where $\partial_{i}$ is the Fourier transform of $p_{i}$. Let $\eta_{i} \in \Omega(M)$ be forms such that $\eta=\sum_{i} \eta_{i} p_{i} \cdot \operatorname{Ind}_{K_{\sigma}}^{K}(\eta)$ is the form on $\operatorname{Ind}_{K_{\sigma}}^{K} M$ whose pull-back to $K \times M$ is

$$
\sum_{i} \pi_{2}^{*} \eta_{i} \wedge \pi_{1}^{*} \phi^{*}\left(\widetilde{\operatorname{curv}}_{K_{\sigma}}^{K}\right)_{i}
$$

Here $\phi: K \rightarrow K / K_{\sigma}$ is the projection. Let $\beta \in \Omega(K \times M)$ be a form which integrates to 1 on the orbits of $K_{\sigma}$ on $K \times M$. Using equation (3.15), we have (omitting pull-backs which confuse the notation)

$$
\begin{aligned}
\left(\mu_{\operatorname{Ind}_{K_{\sigma}}^{K} M, K}\left(\operatorname{Ind}_{K_{\sigma}}^{K}(\eta)\right), h\right)= & \sum_{i, I} \int_{K \times M}\left(\operatorname{Ind}_{K_{\sigma}}^{K} \Phi\right)^{*}\left(\partial_{I} h\right) \exp (\omega) \wedge \eta_{i} \\
& \wedge \operatorname{curv}_{K_{\sigma}, i, I}^{K} \wedge \beta \wedge \exp \left(\operatorname{curv}_{K_{\sigma}}^{K}, \operatorname{Ind}_{K_{\sigma}}^{K} \Phi\right) \\
= & \sum_{i} \int_{M} \Phi^{*}\left(\partial_{i} \operatorname{Vol}_{K_{\sigma}}^{K} \operatorname{Res}_{K_{\sigma}}^{K} h\right) \exp (\omega) \wedge \eta_{i} \\
= & \left(\mu_{M, K_{\sigma}}(\eta), \operatorname{Vol}_{K_{\sigma}}^{K} \operatorname{Res}_{K_{\sigma}}^{K} h\right) \\
= & \left(\operatorname{Ind}_{K_{\sigma}}^{K} \mu_{M, K_{\sigma}}(\eta), h\right)
\end{aligned}
$$

as claimed.

For $\lambda_{0}, \lambda_{1} \in \mathfrak{k}^{*}$ distinct, we say that a distribution $\mu$ on $\mathfrak{k}^{*}$ is smooth, resp. polynomial along the ray $R_{\lambda_{0}, \lambda_{1}}$ at $\lambda \in R_{\lambda_{0}, \lambda_{1}}-\left\{\lambda_{0}\right\}$ if $\mu$ is the induction of a distribution on $\mathfrak{k}_{\lambda}^{*}$ that is polynomial along $R_{\lambda_{0}, \lambda_{1}}$ near $\lambda$. By Proposition 3.7, Proposition 3.6 holds without the assumption that $\lambda_{1}$ is central.

3.5. Comparison of Abelian and non-Abelian DuistermaatHeckman distributions. For the sake of computing examples, it will be helpful to have the formula that compares the Abelian and non-Abelian Duistermaat-Heckman measures. The following result of Harish-Chandra compares Fourier transforms over $\mathfrak{k}$ and $\mathfrak{t}$ : 
Lemma 3.8. For any $h \in \mathcal{S}(\mathfrak{k})^{K}, \Pi \operatorname{Res}_{\mathfrak{t}^{*}}^{\mathfrak{k}^{*}} \mathcal{F}_{\mathfrak{k}}(h)=i^{\operatorname{dim}(K / T) / 2} \mathcal{F}_{\mathfrak{t}}\left(\Pi \operatorname{Res}_{\mathfrak{t}}^{\mathfrak{k}} h\right)$. Proof. Let $\lambda \in \mathfrak{t}^{*}$.

$$
\begin{aligned}
\left(\mathcal{F}_{\mathfrak{k}}(h)\right)(\lambda) & =(2 \pi)^{-\operatorname{dim}(\mathfrak{k} / 2)} \int_{\mathfrak{k}} \mathrm{e}^{2 \pi i(\lambda, \xi)} h(\xi) d \xi \\
& =(2 \pi)^{-\operatorname{dim}(\mathfrak{k} / 2)} \int_{\mathfrak{t}_{+} \times K / T} \mathrm{e}^{2 \pi i(\lambda, k \xi)} h(k \cdot \xi) \exp \left(\omega_{\xi}\right) \frac{\operatorname{RVol}_{T}^{K}(\xi)}{\operatorname{Vol}_{T}^{K}(\xi)} d \xi \\
& =(2 \pi)^{-\operatorname{dim}(\mathfrak{t}) / 2} \int_{\mathfrak{t}_{+}} \sum_{w \in W}(-1)^{l(w)} \frac{h(\xi) \mathrm{e}^{2 \pi i(\lambda, w \xi)} \Pi(\xi)}{\operatorname{Eul}(\mathfrak{k} / \mathfrak{t})(\lambda)} d \xi \\
& =(2 \pi)^{-\operatorname{dim}(\mathfrak{t}) / 2} i^{\operatorname{dim}(\mathfrak{k} / \mathfrak{t}) / 2} \int_{\mathfrak{t}} \frac{h(\xi) \mathrm{e}^{2 \pi i(\lambda, \xi)} \Pi(\xi)}{\Pi(\lambda)} d \xi \\
& =i^{\operatorname{dim}(\mathfrak{k} / \mathfrak{t}) / 2}\left(\Pi^{-1} \mathcal{F}_{\mathfrak{t}}\left(\operatorname{Res}_{T}^{K} h \cdot \Pi\right)\right)(\lambda)
\end{aligned}
$$

As I learned from Paradan, Harish-Chandra's result implies the following relation between Duistermaat-Heckman measures. Note that the Euler class $\operatorname{Eul}(\mathfrak{k} / \mathfrak{t})$ considered as a distribution on $\mathfrak{t}^{*}$ is the product of partial derivatives in the direction of the negative roots of $\mathfrak{k}$.

Theorem 3.9. Let $M$ be a compact Hamiltonian $K$-manifold and $\eta \in$ $\Omega_{K}(M)$ closed. $\mu_{M, K}(\eta)=(\# W)^{-1} \operatorname{Ind}_{T}^{K} \operatorname{Eul}(\mathfrak{k} / \mathfrak{t}) \mu_{M, T}\left(\operatorname{Res}_{T}^{K} \eta\right)$.

Proof. Since $\operatorname{Res}_{T}^{K} \mathcal{F}_{\mathfrak{k}}^{-1} \mu_{M, K}(\eta)=\mathcal{F}_{\mathfrak{t}}^{-1} \mu_{M, T}(\eta)$, we have using Lemma 3.8

$$
\begin{aligned}
\left(\mu_{M, K}(\eta), \mathcal{F}_{\mathfrak{k}}(h)\right) & =\left(\mathcal{F}_{\mathfrak{k}}^{-1}\left(I_{M, K}(\mathcal{L} \wedge \eta)\right), h\right) \\
& =(\# W)^{-1}\left(\operatorname{RVol}_{T}^{K} \mathcal{F}_{\mathfrak{t}}^{-1}\left(I_{M, T}(\mathcal{L} \wedge \eta)\right), \operatorname{Res}_{T}^{K} h\right) \\
& =(\# W)^{-1}\left(I_{M, T}(\mathcal{L} \wedge \eta), \mathcal{F}_{\mathfrak{t}}\left(\operatorname{RVol}_{T}^{K} \operatorname{Res}_{T}^{K} h\right)\right) \\
& =(\# W)^{-1}\left(I_{M, T}(\mathcal{L} \wedge \eta), \mathcal{F}_{\mathfrak{t}}\left(\Pi^{2} \operatorname{Vol}(K / T) \operatorname{Res}_{T}^{K} h\right)\right) \\
& =(\# W)^{-1}\left(\mu_{M, T}(\eta), \operatorname{Eul}(\mathfrak{k} / \mathfrak{t}) \Pi \operatorname{Vol}(K / T) \operatorname{Res}_{T}^{K} \mathcal{F}_{\mathfrak{k}}(h)\right) \\
& =(\# W)^{-1}\left(\mu_{M, T}(\eta), \operatorname{Eul}(\mathfrak{k} / \mathfrak{t}) \operatorname{Vol}_{T}^{K} \operatorname{Res}_{T}^{K} \mathcal{F}_{\mathfrak{k}}(h)\right) .
\end{aligned}
$$

This formula has as a corollary a result of Martin [30], which compares cohomological pairings on the Abelian and non-Abelian quotients. We denote by $M_{T,(\lambda)}$ the symplectic quotients for the action of $T$ :

$$
M_{T,(\lambda)}=\left(\operatorname{Res}_{T}^{K} \Phi\right)^{-1}(\lambda / T) .
$$

Proposition 3.10. If $\lambda$ is a regular value of $\Phi$ and $\operatorname{Res}_{T}^{K} \Phi$, then

$$
I_{M_{(\lambda)}}\left(\kappa_{\lambda}(\eta)\right)=\left(\# W_{\lambda}\right)^{-1} I_{M_{(\lambda), T}}\left(\kappa_{\lambda}\left(\operatorname{Res}_{T}^{K} \eta \wedge \operatorname{Eul}\left(\frac{\mathfrak{k}}{\mathfrak{t}}\right) \wedge \operatorname{Eul}\left(\left(\frac{\mathfrak{k}}{\mathfrak{k}_{\lambda}}\right)^{*}\right)\right)\right) .
$$


Proof. From Theorem (3.9) and Proposition 3.3 (a), we have for generic $\lambda$

$$
I_{M_{(\lambda)}}\left(\kappa_{\lambda}(\eta)\right)=I_{M_{(\lambda), T}}\left(\kappa_{\lambda}\left(\operatorname{Res}_{T}^{K} \eta \wedge \operatorname{Eul}\left(\frac{\mathfrak{k}}{\mathfrak{t}}\right)\right)\right) .
$$

The result for arbitrary $\lambda$ follows from Proposition 3.3 part (c).

3.6. Symplectic vector bundles. Let $M$ be a compact Hamiltonian $K$ manifold and $\pi: E \rightarrow M$ a $K$-equivariant symplectic vector bundle, that is, a vector bundle with structure group $S p(2 n, \mathbb{R})$. We recall from $[\mathbf{1 4}]$ that the total space of $E$ can be given the structure of closed 2-form, equal to $\omega$ on the zero section and non-degenerate in a neighborhood of it: Let $\operatorname{Fr}(E)$ denote the frame bundle of $E$ and $\omega_{F}$ the symplectic form on the fiber $F:=\mathbb{R}^{2 n}$. The action of $\operatorname{Sp}(2 n, \mathbb{R})$ on $F$ is Hamiltonian; we denote by $\phi: F \rightarrow \mathfrak{s p}(2 n, \mathbb{R})^{*}$ the moment map. Let $\alpha \in \Omega^{1}(\operatorname{Fr}(E), \mathfrak{s p}(2 n, \mathbb{R}))$ be a connection 1-form. The 2-form

$$
\pi^{*} \omega+d(\alpha, \phi)+\omega_{F} \in \Omega^{2}(\operatorname{Fr}(E) \times F)
$$

(pull-backs from factors are omitted from the notation) is basic and descends to a closed form $\omega_{E}$ on $E \cong \operatorname{Fr}(E) \times_{S p(2 n, \mathbb{R})} F$ with the required properties. By the symplectic embedding theorem, $\omega_{E}$ is the unique form with these properties up to symplectomorphism on a neighborhood of the zero section. The construction also works equivariantly: If $M$ is a Hamiltonian $K$-manifold and $E$ a $K$-equivariant symplectic vector bundle, let $\Phi_{F}$ denote the moment map for the $K$-action on the fiber $F$. The map

$$
\xi \longmapsto \pi^{*}(\Phi, \xi)+\left(\alpha\left(\xi_{\mathrm{Fr}(E)}\right), \phi\right)
$$

is $\operatorname{Sp}(2 n, \mathbb{R})$-invariant and descends to a moment map $\Phi_{E}: E \rightarrow \mathfrak{k}^{*}$.

Let $U(1)_{\zeta}$ be the one-parameter subgroup generated by a central element $\zeta \in \mathfrak{k}$. Suppose $U(1)_{\zeta}$ acts on $E$ fixing only the zero section with positive weights. In this case, the moment map for the action of $U(1)_{\zeta}$ on the fiber $F$ is a positive-definite quadratic form; it follows that $\left(\Phi_{E}, \zeta\right)$ is proper, so $\Phi_{E}$ is proper as well. For any closed form $\eta \in \Omega_{K}(M)$, localization applied to the total space of $E$ gives

$$
\mu_{E, K}\left(\pi^{*} \eta\right)=\mu_{M, K}\left(\eta \wedge \operatorname{Eul}_{\zeta}^{-1}(E)\right)
$$

Non-compactness of $E$ can be remedied as in $[\mathbf{3 6}, \mathbf{3 8}]$.

Suppose that $U(1)_{\zeta}$ acts on $E$ with both positive and negative (but not zero) weights. Let $E=E_{-} \oplus E_{+}$be the decomposition into positive and negative weight bundles. Let $\pi: E^{\prime} \rightarrow M$ be the symplectic vector bundle obtained from $E$ by reversing the symplectic structure on the sub-bundle $E_{-} \subset E$ on which $U(1)_{\zeta}$ acts with negative weights, so that the orientation of $E^{\prime}$ is $(-1)^{\operatorname{dim}\left(E_{-}\right)}$times the orientation on $E$. Since

$$
\operatorname{Eul}(E)_{\zeta}^{-1}=(-1)^{\operatorname{dim}\left(E^{-}\right)} \operatorname{Eul}\left(E^{\prime}\right)_{\zeta}^{-1},
$$


we have

$$
\mu_{M, K}\left(\eta \wedge \operatorname{Eul}(E)_{\zeta}^{-1}\right)=(-1)^{\operatorname{dim}\left(E^{-}\right)} \mu_{E^{\prime}, K}\left(\pi^{*} \eta\right) .
$$

By Proposition 3.6 applied to $E^{\prime}$, for any $\lambda_{0} \in \mathfrak{k}^{*}$ and non-zero $\lambda_{1} \in \mathfrak{k}_{\lambda_{0}}^{*}$, the distribution $\mu_{M, K}\left(\eta \wedge \operatorname{Eul}(E)_{\zeta}^{-1}\right)$ is polynomial along $R_{\lambda_{0}, \lambda_{1}}$ near $\lambda_{0}$.

3.7. Local normal form. Let $(M, \omega, \Phi)$ be a Hamiltonian $K$-manifold and $y \in \Phi^{-1}(\xi)$. Let $\mathfrak{k}_{y}^{\circ} \subset \mathfrak{k}^{*}$ denote the annihilator of $\mathfrak{k}_{y}$. Let $N$ denote an invariant complement of the tangent space to the orbit $T_{y}(K \cdot y)$. Let $S=$ $N /\left(N^{\omega} \cap N\right)$ denote the quotient of $N$ by the kernel of the symplectic form restricted to $N$, called the symplectic slice at $y$. Let $\omega_{S}$ denote the 2 -form on $S$, and $\Phi_{S}$ the quadratic moment map for the action of $K_{y}$. The $K$-manifold

$$
M_{0}=K \times_{K_{y}}\left(S \oplus\left(\mathfrak{k}_{\xi}^{*} \cap \mathfrak{k}_{y}^{\circ}\right)\right) .
$$

has a Hamiltonian $K$-structure with moment map

$$
\Phi_{0}:[k, s, \nu] \mapsto k \cdot\left(\Phi_{S}(s)+\nu+\xi\right) .
$$

The following is proved in Marle [29] and Guillemin-Sternberg [?]:

Theorem 3.11. There exists an equivariant symplectomorphism $\psi$ of a neighborhood $U$ of $y$ in $M$ with a neighborhood $U_{0}$ of $[1,0,0]$ in $M_{0}$.

\section{The Kirwan-Ness stratification}

Let $(M, \omega)$ be a Hamiltonian $K$-manifold with proper moment map $\Phi$ and $f$ one-half the norm-square of the moment map,

$$
f: M \rightarrow \mathbb{R}, \quad f(m)=\frac{1}{2}(\Phi(m), \Phi(m)) .
$$

In general, $f$ is not a Morse-Bott function. The critical set of $f$ consists of points $m$ fixed by the vector field generated by $\Phi(m)$ :

$$
\operatorname{crit}(f)=\left\{m \in M, \quad\left(\Phi(m)_{M}\right)(m)=0\right\} .
$$

Hence, $\Phi^{-1}(0)$ is a component of $\operatorname{crit}(f)$. For any connected component $C \subset \operatorname{crit}(f)$, the intersection $\Phi(C) \cap \mathfrak{t}_{+}$consists of a single point $\xi$. (See $[\mathbf{2 2}$, $3.15]$ for the case $M$ compact; the case $\Phi$ is proper is similar.) Define

$$
\Xi(M)=\{\xi(C), \quad C \subset \operatorname{crit}(f)\} .
$$

For any $\xi \in \Xi(M)$, let

$$
C_{\xi}=\left\{m \in M, \Phi(K m) \cap \mathfrak{t}_{+}=\xi\right\}
$$

which may be a finite union of connected components. Choose a $K$-invariant almost complex structure on $M$, and consider the corresponding $K$-invariant Riemannian metric. For any $m \in M$, let $\left\{m_{t}, t \in[0, \infty)\right\}$ denote the trajectory of $-\operatorname{grad}(f)$. By Theorem A.6 of the appendix, $m_{t}$ converges to a critical point of $f$ as $t \rightarrow \infty$. For any $\xi \in \Xi(M)$, let $M_{\xi}$ denote the stable 
set of the corresponding critical component $C_{\xi}$, that is, the set of $m$ with limit point in $C_{\xi}$. The Kirwan-Ness stratification is

$$
M=\bigcup_{\xi \in \Xi(M)} M_{\xi}
$$

For each $\xi \in \Xi(M)$, let $U(1)_{\xi}$ denote the one-parameter subgroup generated by $\xi$. Let $Z_{\xi}$ denote the union of components of the fixed point set of $U(1)_{\xi}$ meeting $C_{\xi} \cap \Phi^{-1}\left(\mathfrak{t}_{+}\right), Y_{\xi}$ the set of points in $M$ which flow to $Z_{\xi}$ under $-\operatorname{grad}(\Phi, \xi)$, and $\varphi_{\xi}: Y_{\xi} \rightarrow Z_{\xi}$ the map given by the limit of the flow. By $(4.1)$,

$$
Z_{\xi} \cap \Phi^{-1}(\xi)=C_{\xi} \cap \Phi^{-1}(\xi) .
$$

Let $G$ denote the complexification of $K, K_{\xi}$ and $G_{\xi}$ the stabilizers of $\xi$ under the adjoint action of $K$ and $G$, and $P_{\xi}$ the standard parabolic corresponding to $\xi$. Since $(\Phi, \xi)$ is a Morse-Bott function, $Y_{\xi}$ is a smooth $K_{\xi}$-invariant submanifold. Let $Z_{\xi}^{\circ}$ denote the set of points in $Z_{\xi}$ which flow to $C_{\xi}$ under $-\operatorname{grad}\left(\operatorname{Res}_{Z_{\xi}}^{M} f\right)$, and $Y_{\xi}^{\circ}=\varphi_{\xi}^{-1}\left(Z_{\xi}^{\circ}\right)$. By the stable manifold theorem (see e.g., $[\mathbf{4 1}])$, there exists a diffeomorphism

$$
Y_{\xi}^{\circ}=T_{Z_{\xi}^{\circ}} Y_{\xi}^{\circ},
$$

where $T_{Z_{\xi}^{\circ}} Y_{\xi}^{\circ}$ is the normal bundle of $Z_{\xi}^{\circ}$ in $Y_{\xi}^{\circ}$. The following combines results from Kirwan [22, 4.16, 4.17], Ness [34], and Heinzner-Loose [18].

Theorem 4.1. Let $M$ be a Hamiltonian $K$-manifold with proper moment map, and $\xi \in \Xi(M)$.

a) For a suitable choice of invariant almost complex structure, the stratum $M_{\xi}$ is a smooth invariant submanifold which is identical in a neighborhood of $C_{\xi}$ to $K Y_{\xi}^{\circ}$.

b) Suppose that $M$ is equipped with an invariant Kähler structure. For the metric defined by the structure $M_{\xi}$ is a G-invariant Kähler submanifold, $Y_{\xi}^{\circ}$ is $P_{\xi}$-stable and there exist equivariant diffeomorphisms

$$
K \times_{K_{\xi}} Y_{\xi}^{\circ} \longrightarrow G \times{ }_{P_{\xi}} Y_{\xi}^{\circ} \longrightarrow M_{\xi} .
$$

\section{Localization for the norm-square of the moment map}

Define $\nu_{\xi}:=\left.T_{M_{\xi}} M\right|_{Z_{\xi}} \oplus T_{Z_{\xi}} Y_{\xi}$. In this section, we will prove

Theorem 5.1. Let $M$ be a Hamiltonian $K$-manifold with proper moment map and $\eta \in \Omega_{K}(M)$ closed. The restriction of $\mu_{Z_{\xi}, K_{\xi}}\left(\operatorname{Res}_{Z_{\xi}, K_{\xi}}^{M, K} \eta \wedge\right.$ $\left.\operatorname{Eul}\left(\nu_{\xi}\right)_{\xi}^{-1}\right)$ to a neighborhood of $\xi$ has a unique extension $\mu_{Z_{\xi}^{\circ}, K_{\xi}}\left(\operatorname{Res}_{Z_{\xi}^{\circ}, K_{\xi}}^{M, K} \eta \wedge\right.$ $\left.\operatorname{Eul}\left(\nu_{\xi}\right)_{\xi}^{-1}\right)$ that is polynomial on any ray starting at $\xi$, and

$$
\mu_{M, K}(\eta)=\sum_{\xi \in \Xi(M)} \operatorname{Ind}_{K_{\xi}}^{K} \mu_{Z_{\xi}^{\circ}, K_{\xi}}\left(\operatorname{Res}_{Z_{\xi}^{\circ}, K_{\xi}}^{M, K} \eta \wedge \operatorname{Eul}\left(\nu_{\xi}\right)_{\xi}^{-1}\right) .
$$


Properness of the moment map insures that the sum on the right-hand side of equation (5.1) is locally finite and so well defined. See Section 7 for examples.

5.1. Witten's deformation. Let $M$ be a Hamiltonian $K$-manifold with proper moment map $\Phi, X_{f} \in \operatorname{Vect}(M)$ the Hamiltonian vector field for $f=\frac{1}{2}(\Phi, \Phi), g$ an invariant compatible metric on $M, J$ the associated almost complex structure, and $\alpha$ the invariant 1-form

$$
\alpha(\cdot)=g\left(X_{f}, \cdot\right)=\omega\left(X_{f}, J(\cdot)\right) .
$$

We write

$$
d_{K} \alpha(\xi)=d \alpha+2 \pi i(\phi, \xi), \quad(\phi, \xi):=\iota\left(\xi_{M}\right) \alpha .
$$

Note that

$$
(\phi, \Phi)=g\left(X_{f}, X_{f}\right) \geq 0
$$

and equality holds only if $X_{f}=0$. Define

$$
\omega_{s}:=\omega+s d \alpha, \quad \Phi_{s}:=\Phi+s \phi, \quad \tilde{\omega}_{s}:=\omega_{s}+2 \pi i \Phi_{s} .
$$

Lemma 5.2. $\Phi_{s}$ is proper for all $s \geq 0$.

Proof. By equation (5.2),

$$
\left\|\Phi_{s}\right\|^{2}=\|\Phi\|^{2}+2 s(\Phi, \phi)+s^{2}\|\phi\|^{2} \geq\|\Phi\|^{2} .
$$

Hence, $\left\|\Phi_{s}\right\|^{2} \leq C$ implies $\|\Phi\|^{2} \leq C$ which shows that $\Phi_{s}$ is proper.

Define $\mu_{M, K, s}(\eta) \in \mathcal{D}^{\prime}\left(\mathfrak{k}^{*}\right)^{K}$ by

$$
\left(\mu_{M, K, s}(\eta), h\right)=\sum_{I} \int_{M} \eta_{I} \wedge \exp \left(\omega_{s}\right) \partial_{I} h(\Phi(m)+s \phi(m)) .
$$

Since the cohomology class of $\tilde{\omega}_{s}$ is independent of $s$, so is $\mu_{M, K, s}(\eta)$. Let

$$
U=\bigcup_{\xi \in \Xi(M)} U_{\xi}
$$

be an invariant open neighborhood of $\operatorname{crit}(f) \subset M$, so that

a) $\alpha$ is non-zero on $M-U$;

b) each $U_{\xi}$ is an open neighborhood of $C_{\xi}$;

c) $U_{\xi}$ are pairwise disjoint; and

d) each $U_{\xi}$ intersects only orbit-type strata whose closures intersect $C_{\xi}$.

Any union of sufficiently small neighborhoods $U_{\xi}$ of $C_{\xi}$ has these properties. Since $X_{f}$ is tangent to the $K$-orbits, $\phi$ is non-zero on $M-U$. By equation (5.2), for any $R>0$ there exists an $s(R)$ such that $\|\Phi(m)+s \phi(m)\|>R$ for all $m \in M-U$ and $s>s(R)$. Assuming $h$ has support in a ball of some radius $R(h)$,

$$
\int_{M-U} \sum_{I} \eta_{I} \wedge \exp (\omega+s d \alpha) \partial_{I} h(\Phi(m)+s \phi(m))=0
$$


for $s>s(R(h))$. Let $\mu_{\xi, s}:=\mu_{U_{\xi}, K, s}(\eta)$ denote the distribution defined (5.4) except that integration is over $U_{\xi}$. By equation (5.5), for $s$ sufficiently large

$$
\left(\mu_{M, K}(\eta), h\right)=\sum_{\xi \in \Xi(M)}\left(\mu_{\xi, s}, h\right) .
$$

5.2. The limit distributions. We will show that $\mu_{\xi, s}$ has a distributional limit $\mu_{\xi, \infty}$ as $s \rightarrow \infty$. In most of this section, we will assume that $\xi$ is central, that is, fixed by the coadjoint action of $K$.

Lemma 5.3. (Compare Paradan $[\mathbf{3 6}, 3.8]$ )

a) Let $\left[s_{1}, s_{2}\right]$ and $\lambda \in \mathfrak{k}^{*}$ be such that for all $s \in\left[s_{1}, s_{2}\right], \Phi_{s}\left(\partial U_{\xi}\right)$ does not contain $\lambda$. The restriction of $\mu_{\xi, s}$ to a neighborhood of $\lambda$ is independent of $s \in\left[s_{1}, s_{2}\right]$.

b) $\mu_{\xi, s}$ converges to a limit $\mu_{\xi, \infty}$ as $s \rightarrow \infty$.

Proof. (a) follows from Proposition 3.2. (b) $(\phi, \phi)$ is bounded from below by a positive constant on $\partial U_{\xi}$. By $(5.3),\left(\Phi_{s}, \Phi_{s}\right)$ is bounded from below by a constant that approaches infinity as $s$ does. Hence for any $\lambda \in \mathfrak{k}^{*}$, there exists an $s>0$ such that $\Phi_{s_{1}}\left(\partial U_{\xi}\right)$ does not contain $\lambda$ for $s_{1}>s$. The claim follows from (a).

We say that $\xi \in \Xi(M)$ is minimal if $(\xi, \xi)$ is the minimum value of $(\Phi, \Phi)$.

Lemma 5.4. Suppose that $\xi$ is minimal.

a) $\Phi_{s}\left(\partial U_{\xi}\right)$ does not contain $\xi$ for any $s \in[0, \infty)$;

b) $\mu_{\xi, \infty}$ is equal to $\mu_{\xi, 0}$ in a neighborhood of $\xi$;

c) $\mu_{\xi, \infty}$ is polynomial on any ray beginning at $\xi$.

Proof. (a) $(\xi, \xi)$ is the minimum of $(\Phi, \Phi)$ and $C_{\xi}=\Phi^{-1}(K \xi)$, so $(\Phi, \Phi)>$ $(\xi, \xi)$ on $\partial U_{\xi}$. Hence, $\left(\Phi_{s}, \Phi_{s}\right)=(\Phi, \Phi)+2 s(\Phi, \phi)+s^{2}(\phi, \phi) \geq(\Phi, \Phi)>$ $(\xi, \xi)$ on $\partial U_{\xi}$. (b) follows from (a) and Lemma 5.3 (a). (c) follows as in Proposition 3.6.

Suppose that $\xi$ is not minimal, and let $U_{\xi}^{\prime}$ denote the Hamiltonian $K_{\xi^{-}}$ manifold obtained from flipping the negative weights, as in (3.17), with $\omega^{\prime}$ and $\Phi^{\prime} \mid U_{\xi}^{\prime}$ the new 2 -form and moment map. Since $\left(\Phi^{\prime}, \xi\right) \geq(\xi, \xi), \xi$ is minimal for $U_{\xi}^{\prime}$.

Lemma 5.5. $(\phi, \xi)$ is non-negative in a neighborhood of $C_{\xi}$.

Proof. We have $(\phi(x), \xi)=g\left(X_{f}(x), \xi_{M}(x)\right)$ which equals $\left\|\xi_{M}(x)\right\|^{2}$ plus a function whose second derivative vanishes at $C_{\xi}$. (One can write the function explicitly using the local model (3.18).) The function $\left\|\xi_{M}\right\|^{2}$ is Morse-Bott along $Z_{\xi}$, since $Z_{\xi}$ is a component of the fixed point set of $\xi_{M}$ and $K$ is compact. The Hessian of $(\phi, \xi)$ along $Z_{\xi}$ at $C_{\xi}$ is equal to the Hessian of $\left\|\xi_{M}\right\|^{2}$, which is positive. Hence, $(\phi, \xi)$ is non-negative in a neighborhood of $C_{\xi}$. 
After shrinking $U_{\xi}$ if necessary, we may assume that $(\phi, \xi)$ and $\left(\phi^{\prime}, \xi\right)$ are non-negative on $U_{\xi}$. Let $\Phi_{s}^{\prime}=\Phi^{\prime}+s \phi^{\prime}$ denote the Witten deformation for $U_{\xi}^{\prime}$. Define

$$
\Phi_{s}^{u}\left|U_{\xi}=(1-u) \Phi_{s}\right| U_{\xi}+u \Phi_{s}^{\prime} \mid U_{\xi}
$$

and $\mu_{\xi, s}^{u}(\eta)$ the twisted Duistermaat-Heckman distribution for $\omega_{s}^{u}$, as above.

Proposition 5.6. For any $\lambda \in \mathfrak{k}^{*}$, for $s$ sufficiently large, $\mu_{\xi, s}^{u}$ is independent of $u \in[0,1]$ in a neighborhood of $\lambda$.

Proof. By Proposition 3.2, it suffices to show that $\Phi_{s}^{u}\left(\partial U_{\xi}\right)$ does not contain $\lambda$ for all $u$ and $s$ sufficiently large. Since $\phi=\phi^{\prime}$ on $Z_{\xi}$ and is non-vanishing on $Z_{\xi} \cap \partial U_{\xi}$, there exists a neighborhood $V_{\xi}$ of $Z_{\xi} \cap \partial U_{\xi}$ such that $u \phi+(1-u) \phi^{\prime} \neq$ 0 on $V_{\xi}$ for all $u \in[0,1]$. Hence for $s$ sufficiently large, $\Phi_{s}^{u}\left(V_{\xi}\right)$ does not contain $\lambda$. On the other hand, the complement $V_{\xi}^{c}$ of $V_{\xi}$ is compact and so $(\phi, \xi)$ and $\left(\phi^{\prime}, \xi\right)$ are bounded below on $V_{\xi}^{c}$ by a positive constant. It follows that $\left(\Phi_{s}^{u}, \Phi_{s}^{u}\right)$ is bounded below on $V_{\xi}^{c}$ by a constant which approaches infinity as $s$ does. Hence, $\Phi_{s}^{u}\left(V_{\xi}^{c}\right)$ cannot contain $\lambda$ either.

In the case $\xi$ is central, Proposition 5.6 and Lemma 5.4 (b) imply $\mu_{\xi, \infty}=$ $\mu_{U_{\xi}^{\prime}, K_{\xi}}(\eta)$ in a neighborhood of $\xi$. By equation $(3.17), \mu_{U_{\xi}^{\prime}, K_{\xi}}(\eta)=\mu_{Z_{\xi}, K_{\xi}}(\eta \wedge$ $\left.\operatorname{Eul}\left(\nu_{\xi}\right)_{\xi}^{-1}\right)$ in a neighborhood of $\xi$. Proposition 3.7 completes the proof of Theorem 5.1.

Corollary 5.7. If $M$ is a Hamiltonian $K$-manifold with proper moment map and finite number of orbit-type strata, and $\eta \in \Omega_{K}(M)$ closed, then $\mu_{M, K}(\eta)$ is a tempered distribution.

Proof. If $M$ has a finite number of orbit-type strata, then the sum in Theorem 5.1 is finite. $\mu_{\xi, \infty}$ is tempered for all $\xi \in \Xi(M)$, hence $\mu_{M, K}(\eta)$ is a finite sum of tempered distributions.

\subsection{Further comments.}

a) One-parameter localization 2.1 for central, generic one-parameter subgroups is a special case of localization via the norm-square 5.1. Indeed, let $\mathfrak{z} \subset \mathfrak{k}$ denote the center of $\mathfrak{k}$, and suppose that $\zeta \in \mathfrak{z}$. We can use $\zeta$ to shift the moment map $\Phi_{s}=\Phi+s \zeta$. For sufficiently large $s$, an element $m \in M$ is fixed by $\Phi_{s}(m)$ if and only if it is fixed by $\zeta$. The subsets $Z_{\xi}$ are components of $M^{\zeta}$, and Theorem 5.1 reduces to Theorem 2.1.

b) The statement and proof of Theorem (5.1) are the same in the case that $M$ is a Hamiltonian $K$-orbifold with proper moment map.

\section{Pairing with invariant functions}

Let $M$ be a Hamiltonian $K$-manifold with proper moment map, and $\eta \in$ $\Omega_{K}(M)$ closed. By equation (3.13), for each $\xi \in \Xi(M)$, the contribution 
from $\xi$ to $\left(\mu_{M, K}(\eta), h\right)$ is

$$
\left(\mu_{Z_{\xi}^{\circ}, K_{\xi}}\left(\operatorname{Res}_{Z_{\xi}^{\circ}, K_{\xi}}^{M, K} \eta \wedge \operatorname{Eul}\left(\nu_{\xi}\right)_{\xi}^{-1}\right), \operatorname{Vol}_{K_{\xi}}^{K} \operatorname{Res}_{K_{\xi}}^{K} h\right) .
$$

Hence,

$$
\left(\mu_{M, K}(\eta), h\right)=\sum_{\xi \in \Xi(M)}\left(\mu_{Z_{\xi}^{\circ}, K_{\xi}}\left(\eta \wedge \operatorname{Eul}_{\xi}^{-1}\left(\nu_{\xi}\right)\right), \operatorname{Vol}_{K_{\xi}}^{K} \operatorname{Res}_{K_{\xi}}^{K} h\right) .
$$

Suppose that $\Phi^{-1}(\xi)$ is contained in the principal orbit-type stratum for the action of $K_{\xi}$ on $Z_{\xi}$. In this section, we show that the contribution from $\xi$ can be expressed as an integral over the symplectic quotient

$$
Z_{(\xi)}:=\left(Z_{\xi}\right)_{(\xi)}=K \backslash C_{\xi} .
$$

Let $K_{\xi}^{\prime}$ denote the identity component of the generic stabilizer of $K_{\xi}$ on $\Phi^{-1}(\xi) \cap Z_{\xi}$. The assumption that $\Phi^{-1}(\xi) \cap Z_{\xi}$ is contained in the principal orbit-type stratum of $Z_{\xi}$ implies that $K_{\xi}^{\prime}$ acts trivially on the annihilator of $\mathfrak{k}_{\xi}^{\prime}$. It follows that $K_{\xi}^{\prime}$ is normal and the quotient $K_{\xi}^{\prime \prime}:=K_{\xi} / K_{\xi}^{\prime}$ is a compact connected Lie group. Let $K_{\xi, Z_{\xi}}^{\prime \prime}$ denote the (finite) generic stabilizer of $K_{\xi}^{\prime \prime}$ on $Z_{\xi}$. Let $\kappa_{\xi}$ denote the composition of the restriction $H_{K}(M) \rightarrow$ $H_{K_{\xi}}\left(\Phi^{-1}(\xi)\right)$ with the isomorphism

$$
H_{K_{\xi}}\left(\Phi^{-1}(\xi) \cap Z_{\xi}\right) \rightarrow H_{K_{\xi}^{\prime}}\left(Z_{(\xi)}\right)=H\left(Z_{(\xi)}\right) \otimes S\left(\mathfrak{k}_{\xi}^{\prime, *}\right)^{K_{\xi}^{\prime}} .
$$

This extends to forms with smooth coefficients. In particular, any $h \in$ $\mathcal{S}\left(\mathfrak{k}_{\xi}^{*}\right)^{K_{\xi}}$ defines a characteristic class $\kappa_{\xi}(h) \in \mathcal{H}_{K_{\xi}^{\prime}}\left(Z_{(\xi)}\right)$. We denote by

$$
\nu_{(\xi)}:=K_{\xi}^{\prime} \backslash\left(\left.\nu_{\xi}\right|_{\Phi^{-1}(\xi)}\right) \longrightarrow Z_{(\xi)}
$$

the quotient bundle.

Theorem 6.1. If $\Phi^{-1}(\xi) \cap Z_{\xi}$ is contained in the principal orbit-type stratum in $Z_{\xi}$, then equation (6.1) is equal to

$$
\operatorname{Vol}\left(\frac{K_{\xi}^{\prime \prime}}{K_{\xi, Z_{\xi}}^{\prime \prime}}\right) \int_{Z_{(\xi)} \times \mathfrak{k}_{\xi}^{\prime}} \mathcal{L}_{(\xi)} \wedge \operatorname{Eul}\left(\nu_{(\xi)}\right)_{\xi}^{-1} \wedge \kappa_{\xi}\left(\eta \wedge \operatorname{Vol}_{K_{\xi}}^{K} \operatorname{Res}_{K_{\xi}}^{K} h\right) .
$$

Note that the inverted Euler class has tempered-distributional coefficients, while the remaining factor has coefficients in the ring of Schwartz functions. The integral over $\mathfrak{k}_{\xi}^{\prime}$ refers to the pairing of these coefficient rings.

Proof. Suppose that $\Xi(M)=\{0\}, \eta=1, M$ is maximal rank, and $\Phi^{-1}(0)$ is contained in the principal orbit-type stratum for $M$. Let $\alpha \in \Omega^{1}\left(\Phi^{-1}(0), \mathfrak{k}\right)^{K}$ denote a connection 1-form for the action of $K$ on $\Phi^{-1}(0)$. Let $\pi_{0}: \Phi^{-1}(0) \rightarrow$ $M_{(0)}$ denote the projection. By the co-isotropic embedding theorem, a neighborhood of $\Phi^{-1}(0)$ is $K$-symplectomorphic to a neighborhood of $\Phi^{-1}(0) \times\{0\}$ in the Hamiltonian $K$-manifold

$$
\left(\Phi^{-1}(0) \times \mathfrak{k}^{*}, \quad \pi_{0}^{*} \omega_{(0)}+d(\lambda, \alpha)\right) .
$$


Let $\pi_{1}$ and $\pi_{2}$ denote the projections

$$
\Phi^{-1}(0) \stackrel{\pi_{1}}{\rightarrow} T \backslash \Phi^{-1}(0) \stackrel{\pi_{2}}{\longrightarrow} M_{(0)} .
$$

The 2 -form $d \alpha$ is $T$-basic and descends to a closed $\mathfrak{k}$-valued 2 -form $\pi_{1, *} d \alpha$. By equation (6.3) and Section 3.3, the volumes of quotients at generic $\lambda \in \mathfrak{k}^{*}$ are

$$
p(\lambda):=\operatorname{Vol}\left(M_{(\lambda)}\right)=I_{T \backslash \Phi^{-1}(0)}\left(\exp \left(\pi_{2}^{*} \omega_{(0)}+\left(\lambda, \pi_{1, *} d \alpha\right)\right)\right) .
$$

Let $h \in \mathcal{S}\left(\mathfrak{k}^{*}\right)^{K}, f=\mathcal{F}_{\mathfrak{t}}^{-1}\left(\Pi \operatorname{Re}_{\mathfrak{t}^{*}}^{\mathfrak{k}^{*}} h\right)$ and $p=\sum_{I} p_{I} \lambda_{I}$. Using equation $(3.10)$

$$
\begin{aligned}
\operatorname{Vol}\left(K / K_{M}\right)^{-1}\left(\mu_{M, K}, h\right) & =\left(\left(\operatorname{Vol}_{T}^{K}\right)^{-1} p, h\right)_{\mathfrak{k}^{*}} \\
& =(\# W)^{-1}\left(\operatorname{Res}_{\mathfrak{t}^{*}}^{\mathfrak{k}^{*}} p, \Pi h\right)_{\mathfrak{t}^{*}} \\
& =(\# W)^{-1}\left(\mathcal{F}_{\mathfrak{t}}^{-1}\left(\operatorname{Res}_{\mathfrak{t}^{*}}^{\mathfrak{k}^{*}} p\right), \mathcal{F}_{\mathfrak{t}}^{-1}\left(\Pi \operatorname{Res}_{\mathfrak{t}^{*}}^{\mathfrak{k}^{*}} h\right)\right)_{\mathfrak{t}} \\
& =(\# W)^{-1} \sum_{I} p_{I}\left(\partial_{I} f\right)(0) \\
& =(\# W)^{-1} I_{T \backslash \Phi^{-1}(0)}\left(\exp \left(\pi_{2}^{*} \omega_{(0)}\right) f\left(\pi_{1, *} \frac{d \alpha}{2 \pi i}\right)\right)
\end{aligned}
$$

defined using the formal power series of $f$ at 0 . Choose an orthonormal basis $\xi_{1}, \ldots, \xi_{r}$ for $\mathfrak{t}$ and $\xi_{r+1}, \ldots, \xi_{n}$ for $\mathfrak{k} / \mathfrak{t} \cong \mathfrak{t}^{\perp}$. We can replace the integral over $T \backslash \Phi^{-1}(0)$ with

$$
\frac{1}{\operatorname{Vol}(T)} \int_{\Phi^{-1}(0)}\left(\exp \left(\pi_{2}^{*} \omega_{(0)}\right) \wedge f\left(\frac{d \alpha}{2 \pi i}\right) \wedge \prod_{j=1}^{r}\left(\alpha, \xi_{j}\right)\right) .
$$

It remains to integrate over the fiber $K$ of $\Phi^{-1}(0) \rightarrow M_{(0)}$. Writing

$$
d \alpha=\pi_{0}^{*} \operatorname{curv}(\alpha)-\frac{1}{2}[\alpha, \alpha]
$$

One sees that the component of $f\left(\frac{d \alpha}{2 \pi i}\right) \wedge \prod_{j=1}^{r}\left(\alpha, \xi_{j}\right)$ that contributes to the fiber integral is

$$
\left(\mathcal{F}_{\mathfrak{t}}^{-1} \Pi \star f\right)\left(\pi_{0}^{*} \frac{\operatorname{curv}(\alpha)}{2 \pi i}\right) \wedge \prod_{i=1}^{n}\left(\alpha, \xi_{j}\right) .
$$

Integrating over the fiber changes the factor $\prod_{i=1}^{n}\left(\alpha, \xi_{j}\right)$ to $\operatorname{Vol}(K)$. We have

$$
\begin{aligned}
(\# W)^{-1}\left(\mathcal{F}_{t}^{-1}\left(\Pi^{2} \operatorname{Res}_{\mathfrak{t}^{*}}^{\mathfrak{k}^{*}} h\right)\right)\left(\frac{\operatorname{curv}(\alpha)}{2 \pi i}\right) & =\left(\mathcal{F}_{\mathfrak{k}}^{-1}(h)\right)\left(\frac{\operatorname{curv}(\alpha)}{2 \pi i}\right) \\
& =\mathcal{L}_{0} \wedge \kappa_{0}(h) .
\end{aligned}
$$

It follows that

$$
\left(\mu_{M, K}, h\right)=\operatorname{Vol}\left(K / K_{M}\right) I_{M_{(0)}}\left(\mathcal{L}_{0} \wedge \kappa_{0}(h)\right) .
$$


The general case is the similar and left to the reader except that the forms on $Z_{(\xi)}$ are $K_{\xi}^{\prime}$-equivariant and the form $\eta$ is to be included.

Corollary 6.2. Let $M$ be a compact Hamiltonian $K$-manifold, $\eta \in \Omega_{K}(M)$ closed, and $h \in \mathcal{S}\left(\mathfrak{k}^{*}\right)^{K}$. Suppose that $\Phi^{-1}(\xi) \cap Z_{\xi}$ is contained in the principal orbit-type stratum of $Z_{\xi}$, for all $\xi \in \Xi(M)$. The pairing $\left(I_{M, K}(\eta), \mathcal{F}_{\mathfrak{k}}^{-1}(h)\right)$ is equal to

$$
\sum_{\xi \in \Xi(M)} \operatorname{Vol}\left(\frac{K_{\xi}^{\prime \prime}}{K_{\xi, Z_{\xi}}^{\prime \prime}}\right) \int_{Z_{(\xi)} \times \mathfrak{k}_{\xi}^{\prime}} \kappa_{\xi}\left(\eta \wedge \operatorname{Vol}_{K_{\xi}}^{K} \operatorname{Res}_{K_{\xi}}^{K} h\right) \wedge \operatorname{Eul}\left(\nu_{(\xi)}\right)_{\xi}^{-1} .
$$

Proof. Consider the family of equivariant symplectic forms $\epsilon \omega_{K}$ for $\epsilon \in(0,1]$. The corollary follows from taking the limit $\epsilon \rightarrow 0$ of equation (6.2), using Theorem 6.1: the stratification is independent of $\epsilon$, and $\mathcal{L}_{(\xi)} \rightarrow 1$ as $\epsilon \rightarrow 0$.

If $\Phi^{-1}(\xi) \cap Z_{\xi}$ is not contained in the principal orbit-type stratum of $Z_{\xi}$, then equation (6.1) can be written as a finite sum of integrals over symplectic quotients near $\xi$, by the gluing rule in Meinrenken [33], but I do not know a nice formula for the limit.

\section{Examples}

In these examples, we will compare the one-parameter and norm-square localization formulas.

7.1. $\mathbf{K}=\mathbf{U}(\mathbf{1})$ acting on $\mathbf{M}=\mathbb{P}^{\mathbf{1}}$. We identify the Lie algebra $i \mathbb{R}$ of $U(1)$ with $\mathbb{R}$ by division by $2 \pi i$. If we choose on $\mathbb{R}$ the standard inner product, then the weight lattice becomes identified with $\mathbb{Z}, \mu_{\mathfrak{k}^{*}}$ is Lebesgue measure on $\mathbb{R}$, and the volume of $K$ is 1 . The action of $U(1)$ on $\mathbb{P}^{1}$ by $z\left[w_{0}, w_{1}\right]=\left[z^{-a} w_{0}, z^{-b} w_{1}\right]$ has moment map

$$
\Phi\left(\left[w_{0}, w_{1}\right]\right)=\frac{a\left|w_{0}\right|^{2}+b\left|w_{1}\right|^{2}}{\left|w_{0}\right|^{2}+\left|w_{1}\right|^{2}} .
$$

There are two fixed points, at $w_{0}=0$, resp. $w_{1}=0$. The tangent weights at the fixed points are $\pm(b-a)$. Let $H_{ \pm}$denote the Heaviside distributions, equal to $\mu_{\mathfrak{k}^{*}}$ on the positive (resp. negative) real numbers and zero elsewhere. One-parameter localization with $\xi>0$ gives

$$
\mu_{M, K}=\frac{1}{b-a}\left(\delta(a) \star H_{+}-\delta(b) \star H_{+}\right)=\frac{\chi_{[a, b]} \mu_{\mathfrak{k}^{*}}}{b-a},
$$

where $\chi_{[a, b]}$ the characteristic function for the interval $[a, b]$ and $\star$ denotes convolution. For negative action chamber $\xi<0$, localization gives

$$
\mu_{M, K}=\frac{1}{b-a}\left(-\delta(a) \star H_{-}+\delta(b) \star H_{-}\right)=\frac{\chi_{[a, b]} \mu_{\mathfrak{k}^{*}}}{b-a} .
$$

This is shown graphically in Figure 1. 


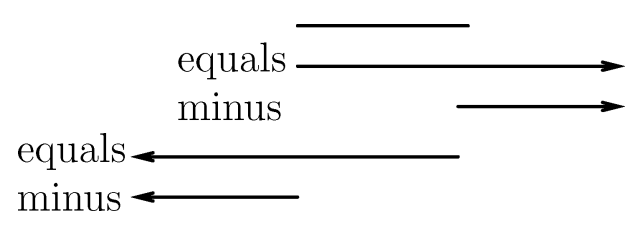

Figure 1. One-parameter localization for $\mathbb{P}^{1}$.

The Kirwan-Ness localization for $a<0<b$ is as follows. The critical components of $f$ are the two $T$-fixed points and the zero level set:

$$
\begin{gathered}
C_{a}=\left\{w_{0}=0\right\}=M_{a}, \quad C_{b}=\left\{w_{1}=0\right\}=M_{b} \\
C_{0}=\Phi^{-1}(0), \quad M_{0}=\left\{\left[w_{1}, w_{2}\right], w_{1} w_{2} \neq 0\right\}=M-M_{a}-M_{b} .
\end{gathered}
$$

For $\xi=0, Z_{\xi}^{\circ}=M_{\xi}$ is the complement of the $K$-fixed points. The Duistermaat-Heckman measure for $Z_{\xi}^{\circ}$ is $\mu_{\mathfrak{k}^{*}} \chi_{[a, b]} /(b-a)$. Its unique extension which is piecewise polynomial on any ray beginning at 0 is $\mu_{\mathfrak{k}^{*}} /(b-a)$. Theorem 5.1 gives

$$
\begin{aligned}
\mu_{M, K} & =\mu_{M, 0}+\mu_{M, b}+\mu_{M, a} \\
& =\frac{1}{b-a}\left(\mu_{\mathfrak{k}^{*}}-H_{-} \star \delta(a)-H_{+} \star \delta(b)\right) \\
& =\frac{1}{b-a} \mu_{\mathfrak{k}^{*}}\left(1-\chi_{(-\infty, a]}-\chi_{[b, \infty)}\right) .
\end{aligned}
$$

The formula is shown graphically in Figure 2.

7.2. $\mathbf{S U}(3)$ acting on $\mathbf{a} \mathbf{G}_{\mathbf{2}}$-coadjoint orbit. In this example, we apply the localization formulas to the action of $S U(3) \subset G_{2}$ on a coadjoint orbit of $G_{2}$. Let $K=S U(3)$, and $\omega_{1}$ and $\omega_{2}$ the fundamental weights. Let $G_{2}$ denote the connected simple complex group of type $G_{2}$. The dual positive Weyl chamber for $G_{2}$ is the span of $\omega_{1}$ and $\omega_{1}+\omega_{2}$. Let $P_{\omega_{1}+\omega_{2}}$ denote the maximal parabolic of $G_{2}$, so that $M=G_{2} / P_{\omega_{1}+\omega_{2}}$ is diffeomorphic to the co-adjoint orbit through $\omega_{1}+\omega_{2}$. The Weyl group $W$ for $S U(3)$ acts simply transitively on the $T$-fixed points. First, we compute the DuistermaatHeckman measure using ordinary localization. The contribution to $\mu_{M, T}$ from the fixed point $x(w)$ corresponding to $w \in W$ is

$$
\delta_{w \mu} \star \prod_{j=1}^{5} \pm H_{ \pm w \beta_{j}},
$$

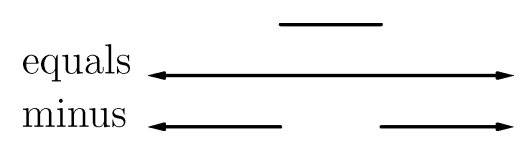

Figure 2. Norm-square localization for $\mathbb{P}^{1}$. 


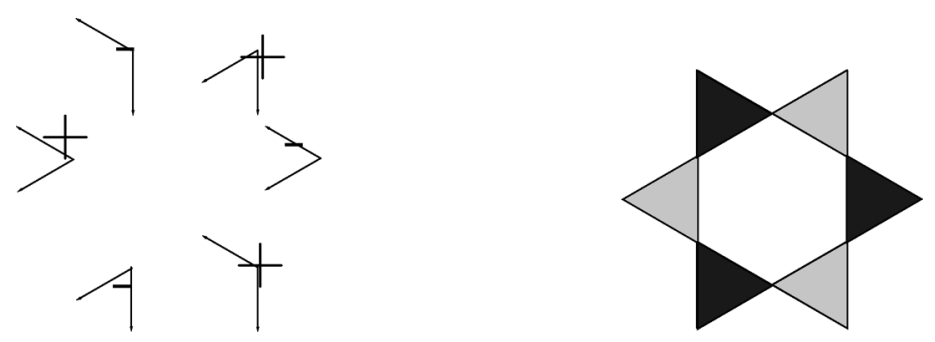

Figure 3. One-parameter localization for $G_{2} / P$.

where $\beta_{j}$ are the positive roots of $G_{2}$ not vanishing at $\omega_{1}+\omega_{2}$

$$
2 \omega_{1}-\omega_{2},-\omega_{1}+2 \omega_{2}, \omega_{1}+\omega_{2}, 3 \omega_{1}, 3 \omega_{2}
$$

and the signs are determined by the action chamber. The $\beta_{j}$ that are not roots of $S U(3)$ are $\beta_{5}=3 \omega_{1}, \beta_{6}=3 \omega_{2}$. By Theorem 3.9

$$
\mu_{M, K}=\frac{1}{6} \operatorname{Ind}_{T}^{K} \sum_{w \in W}(-1)^{l(w)} \delta_{w\left(\omega_{1}+\omega_{2}\right)} \star\left( \pm H_{ \pm 3 w \omega_{1}}\right) \star\left( \pm H_{ \pm 3 w \omega_{2}}\right) .
$$

The contributions are shown in Figure 3. Each contribution is $\pm \mu_{\mathrm{t}^{*}} /$ $\left(9\left\|\omega_{1}\right\|\left\|\omega_{2}\right\|\right)$ where non-negative. Positive (resp. negative) contributions are shown in light (resp. dark) shading. The moment polytope for $M$ is

$$
P=\operatorname{hull}\left(\omega_{1}, \omega_{2}, \omega_{1}+\omega_{2}\right) .
$$

Let $F_{1}$ be the open face connecting $\omega_{2}, \omega_{1}+\omega_{2}, F_{2}$ the open face connecting $\omega_{1}, \omega_{1}+\omega_{2}$, and $F_{3}$ the open face connecting $\omega_{1}, \omega_{2}$. Let $F_{i j}=F_{i} \cap F_{j}$.

We compute the Kirwan-Ness stratification. The inverse image $\Phi^{-1}\left(F_{12}\right)$ contains a unique point, $x(1) \in M$, which is $T$-fixed. None of the other $T$ fixed points map to $\mathfrak{t}_{+}^{*}$. Therefore, the remaining points in $\Phi^{-1}\left(\operatorname{int}\left(\mathfrak{t}_{+}^{*}\right)\right)$ have one-dimensional stabilizers. Since $\Phi^{-1}\left(\operatorname{int}\left(\mathfrak{t}_{+}^{*}\right)\right)$ has $\operatorname{dimension} 2 \operatorname{dim}(T)$, it is a toric manifold, so the inverse image of any face $F \subset$ int $\mathfrak{t}_{+}^{*}$ has infinitesimal stabilizer the annihilator of the tangent space of $F$. The stabilizers of the faces $F_{1}, F_{2}$, and $F_{3}$ are

$$
\mathfrak{t}_{1}=\operatorname{span}\left(h_{1}\right), \quad \mathfrak{t}_{2}=\operatorname{span}\left(h_{2}\right), \quad \mathfrak{t}_{3}=\operatorname{span}\left(h_{3}\right),
$$

where $h_{1}, h_{2}$, and $h_{3}$ are the coroots of $S U(3)$. The level set $\Phi^{-1}\left(\left(\omega_{1}+\omega_{2}\right) / 2\right)$ is critical with $\xi=\left(\omega_{1}+\omega_{2}\right) / 2$. The fixed point component $Z_{\xi}$ has moment image

$$
\Phi\left(Z_{\xi}\right)=\operatorname{hull}\left(2 \omega_{2}-\omega_{1}, 2 \omega_{1}-\omega_{2}\right) .
$$

The unstable manifold $Y_{\xi}$ has image under the moment map for $T$

$$
\operatorname{proj}_{\mathfrak{t}}^{\mathfrak{k}} \Phi\left(\overline{Y_{\xi}}\right)=\operatorname{hull}\left(2 \omega_{2}-\omega_{1}, 2 \omega_{1}-\omega_{2}, \omega_{1}+\omega_{2}\right) .
$$

None of the other faces $F_{j}$ contain points $\xi$ with $\xi \in \mathfrak{t}_{j}$. Therefore, there are no other critical points in $\Phi^{-1}\left(\operatorname{int}\left(\mathfrak{t}_{+}^{*}\right)\right)$. Finally, consider the inverse image 


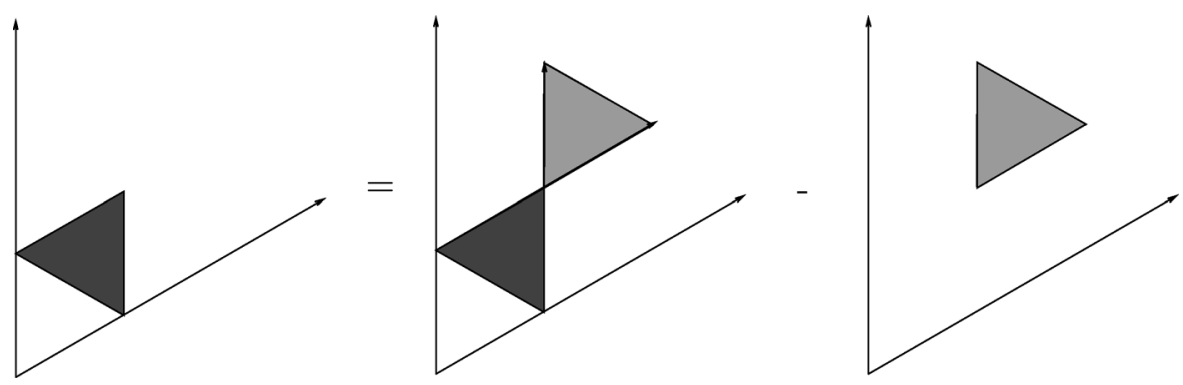

Figure 4. Norm-square localization for $G_{2} / P$.

of the vertices $F_{j k}=F_{13}$ or $F_{23} . \Phi^{-1}\left(F_{j k}\right)$ does not contain a $T$-fixed point. $\Phi^{-1}\left(F_{j k}\right)$ does not contain a point $m$ stabilized by $\operatorname{span}\left(F_{j k}\right)$. Indeed, since the stabilizer $K_{m}$ does not contain a maximal torus, $K_{m}$ cannot intersect the semisimple part $\left[K_{\Phi(m)}, K_{\Phi(m)}\right]$. Therefore, $K_{m}$ is one-dimensional. Let $X$ denote the fixed point component of $K_{m}$ containing $m$. Since $K_{m}$ is onedimensional, the image $\Phi(X)$ is codimension one, and so meets $\Phi^{-1}\left(\operatorname{int}\left(t_{+}^{*}\right)\right)$. This implies that the $\mathfrak{k}_{m}$ is conjugate to either $\mathfrak{t}_{j}$ or $\mathfrak{t}_{k}$, and so $\mathfrak{k}_{m}$ cannot equal the span of $F_{j k}$. Therefore,

$$
\Xi(M)=\left\{\omega_{1}+\omega_{2}, \frac{1}{2}\left(\omega_{1}+\omega_{2}\right)\right\} .
$$

One can show that the Kirwan-Ness stratification coincides with the orbit stratification for $G$, just as in the previous example. In particular, $M$ is a two-orbit variety, with one open orbit and one of complex codimension two.

The contributions to the norm-square localization formula can be described as follows. For $\xi=\left(\omega_{1}+\omega_{2}\right) / 2$, we have

$$
\mu_{\xi, \infty}=\delta\left(\left(\omega_{1}+\omega_{2}, \xi\right)=\frac{1}{2}\left\|\omega_{1}+\omega_{2}\right\|^{2}\right) \star \frac{H_{\omega_{1}+\omega_{2}}}{\left(9\left\|\omega_{1}\right\|\left\|\omega_{2}\right\|\right)},
$$

where $H_{\omega_{1}+\omega_{2}}$ is the Heaviside distribution for $\left(\omega_{1}+\omega_{2}, \xi\right) \geq 0$. Therefore,

$$
\operatorname{Ind}_{T}^{K} \mu_{\xi}^{\infty}=\operatorname{Ind}_{T}^{K} \frac{\left(\chi_{P}-\chi_{Q}\right) \mu_{\mathfrak{t}^{*}}}{\left(9\left\|\omega_{1}\right\|\left\|\omega_{2}\right\|\right)}
$$

where $\chi_{P}$ and $\chi_{Q}$ are the characteristic functions for the polytope $P$, resp. the cone

$$
Q=\mathbb{R}_{\leq 0}\left(P-\left(\omega_{1}+\omega_{2}\right)\right)+\omega_{1}+\omega_{2} .
$$

For $\xi=\omega_{1}+\omega_{2}$, we get

$$
\operatorname{Ind}_{T}^{K} \mu_{\xi}^{\infty}=\operatorname{Ind}_{T}^{K} \frac{\chi_{Q} \mu_{\mathrm{t}^{*}}}{9\left\|\omega_{1}\right\|\left\|\omega_{2}\right\|} .
$$

Hence,

$$
\mu_{M, K}=\operatorname{Ind}_{T}^{K}\left(\frac{\chi_{P} \mu_{\mathrm{t}^{*}}}{9\left\|\omega_{1}\right\|\left\|\omega_{2}\right\|}\right)
$$


see Figure 4.

\section{A remark on sheaf cohomology}

In algebraic geometry, there is a formula which expresses the index of a sheaf of a stratified variety as a sum over the strata. Let $G$ be a reductive complex group, $R(G)$ the ring of finite linear combinations of irreducible characters, and $\mathcal{R}(G)=\operatorname{Hom}(R(G), \mathbb{Z})$ its dual. Let $M$ be a smooth variety and $E \rightarrow M$ a $G$-equivariant vector bundle. The equivariant index of $E$ is the virtual representation

$$
I_{M, K}(E)=\sum_{j=0}^{\operatorname{dim}(M)}(-1)^{j} H^{j}(M, E) .
$$

We will assume that the multiplicity of any irreducible representation is finite, so that $I_{M, K}(E)$ defines an element in $\mathcal{R}(G)$.

Suppose $M$ decomposes into a disjoint union of smooth $G$-stable subvarieties

$$
M=\bigcup_{\xi \in \Xi(M)} M_{\xi}
$$

Let $T_{M_{\xi}} M$ is the normal bundle of $M_{\xi} \rightarrow M, T_{M_{\xi}}^{*} M$ its dual. The Euler class

has a formal inverse

$$
\operatorname{Eul}\left(T_{M_{\xi}} M\right):=\Lambda^{\text {even }}\left(T_{M_{\xi}} M\right) \ominus \Lambda^{\text {odd }}\left(T_{M_{\xi}} M\right)
$$

$$
\operatorname{Eul}\left(T_{M_{\xi}} M\right)^{-1}:=(-1)^{\operatorname{codim}\left(M_{\xi}\right)} \operatorname{det}\left(T_{M_{\xi}}^{*} M\right) \otimes S\left(T_{M_{\xi}}^{*} M\right),
$$

where $S$ resp. $\Lambda$ denotes the direct sum of symmetric resp. exterior powers and det the top exterior power. Let $\operatorname{Res}_{M_{\xi}}^{M}$ denote restriction to $M_{\xi}$. The Cousin-Grothendieck spectral sequence (take the Euler characteristic of the local cohomologies) produces a formula in $\mathcal{R}(G)$

$$
I_{M, K}(E)=\sum_{\xi \in \Xi(M)} I_{M_{\xi}, K}\left(\operatorname{Res}_{M_{\xi}}^{M} E \otimes \operatorname{Eul}\left(T_{M_{\xi}} M\right)^{-1}\right)
$$

assuming that the representations on the right-hand side have finite multiplicities, see Teleman [43] and Hartshorne [17, Section 4].

In some sense, the localization theorems in equivariant cohomology or $K$-theory are attempts to extend this result to manifolds with group actions; so far, this has been done only in special cases. One parameter localization arises from the stratification defined by the action of a circle subgroup $G=\mathbb{C}^{*}$. Let $\Xi(M)$ denote the set of connected components of the fixed point set $M^{G}$ in $M$. For any $\xi \in \Xi(M)$, let $M_{\xi}=\left\{m \in M, \lim _{z \rightarrow 0} z m \in \xi\right\}$ denote the stable manifolds for the flow generated by the action, as in BialinickiBirula [6]. The formula Theorem (8.1) is a sheaf-theoretic version of Theorem 2.1. Equation (8.1) applied to the Kirwan-Ness stratification gives a 
sheaf-theoretic version of Theorem 5.1. It remains an open question, at least for me, whether there is a more general formula in equivariant $K$-theory or in equivariant de Rham theory analogous to equation (8.1). For instance, the decomposition of a spherical $G$-variety into $G$-orbits produces a formula in $K$-theory not covered by one-parameter localization or localization via the norm-square of the moment map.

\section{Two-dimensional Yang-Mills}

The basic reference for mathematical two-dimensional Yang-Mills theory is Atiyah-Bott [1] . Let $K$ denote a connected compact Lie group and $G$ the complexification of $K$. Fix the basic inner product $():, \mathfrak{k} \times \mathfrak{k} \rightarrow \mathbb{R}$ and use it to identify $\mathfrak{k}$ with its dual $\mathfrak{k}^{*}$. Let $P$ be a principal $K$-bundle and $P(\mathfrak{k})=P \times_{K} \mathfrak{k}$ the adjoint bundle. Similarly, let $P(G)=P \times_{K} G$ the associated principal $G$-bundle. Let $\Omega^{\bullet}(X, P(\mathfrak{k}))$ the space of forms with values in $P(\mathfrak{k})$. The inner product on $\mathfrak{k}$ induces a metric on $P(\mathfrak{k})$. Combining this with the wedge product gives map

$$
\Omega^{k}(X, P(\mathfrak{k})) \times \Omega^{l}(X, P(\mathfrak{k})) \rightarrow \Omega^{k+l}(X), \quad\left(a_{1}, a_{2}\right) \mapsto\left(a_{1} \wedge a_{2}\right) .
$$

Choose a metric on $X$ and let $*$ denote the associated Hodge star operator $\Omega^{k}(X, P(\mathfrak{k})) \rightarrow \Omega^{2-k}(X, P(\mathfrak{k}))$. Let

$$
\mathcal{A}(P)=\Omega^{1}(X, P(\mathfrak{k}))
$$

the affine space of connections and

$$
K(P)=\operatorname{Aut}_{K}(P), \quad G(P)=\operatorname{Aut}_{G}(P(G))
$$

the group of unitary, resp. complex gauge transformations. For any $A \in$ $\mathcal{A}(P)$, let $F_{A} \in \Omega^{2}(X, P(\mathfrak{k}))$ denote its curvature. Yang-Mills theory is the area-dependent quantum field theory with partition function given

$$
Z(X)=\sum_{P} Z(P)
$$

where the sum is over isomorphism classes of principal $K$-bundles $P$ and $Z(P)$ is defined formally by the path integral

$$
" Z(P)=\frac{1}{\operatorname{Vol}(K(P))} \int_{\mathcal{A}(P)} \exp (-S(A)) D A ", \quad S(A)=\frac{1}{2 \epsilon} \int_{X}\left(F_{A} \wedge * F_{A}\right) .
$$

Formally, $Z(P)$ is the pairing of the Duistermaat-Heckman measure for the action of $K(P)$ on $\mathcal{A}(P)$ with a Gaussian on $\Omega^{2}(X, P(\mathfrak{k}))$.

A definition of the two-dimensional Yang-Mills integral, including observables, is given by Lévy [27]. Lévy's approach is to embed the space of connections mod gauge equivalence into the space of maps of the loop space on $\Sigma$ to the group mod conjugacy via the holonomy map. Lévy constructs a probability measure on this "thickening" of the space of connections and proves that the Yang-Mills integral is given by the Migdal formula. 
Here, we will define the Yang-Mills integral by assuming that localization for the norm-square (5.1) holds. The strategy of defining path integrals by expanding over critical components of the integrand appears in many places, such as perturbative Chern-Simons theory [3]. The purpose of this section is to show that with this definition, the Yang-Mills integral is given by the Migdal formula, and hence agrees with Lévy's definition. This might be seen as an easy two-dimensional analog of the much harder conjecture regarding the three-dimensional Chern-Simons path integral, that the "exact" definition via Reshetikhin-Turaev agrees with the "perturbative" definition of Axelrod-Singer.

The action of $K(P)$ on $\mathcal{A}(P)$ is Hamiltonian with moment map minus the curvature, and so the Yang-Mills function $S(A)$ is the norm-square of the moment map. The critical points of $S(A)$ are the connections satisfying the Yang-Mills equation

$$
d_{A}^{*} F_{A}=0 .
$$

These are the connections with constant central curvature. Each $* F_{A}(x)$ lies in an orbit of $K$ on $P(\mathfrak{k})_{x}$, parametrized by some $\xi \in \mathfrak{t}_{+}$independent of $x \in X$. In the case $K=U(r), P$ is the principal $U(r)$ bundle of rank $r$ and degree $d$ over a surface $X$ of genus at least one,

$$
\Xi(P)=\left\{\left(\mu_{1}, \ldots, \mu_{1}, \mu_{2}, \ldots, \mu_{2}, \ldots, \mu_{r}\right)\right\} \subset \mathbb{Q}^{r}
$$

the set of non-increasing sequences such that $\mu_{j}=d_{j} / r_{j}$ for some integers $d_{j}$ and $r_{j}$ such that $\sum_{j} d_{j}=d, \sum_{j} r_{j}=r$ and each $\mu_{j}$ appears $r_{j}$ times. If $X$ is genus zero, then only integral $\mu_{j}$ appear.

Minus the gradient flow of $S(A)$ induces a decomposition of $\mathcal{A}(P)$ into stable manifolds

$$
\mathcal{A}(P)=\bigcup_{\xi \in \Xi} \mathcal{A}(P)_{\xi}
$$

By the results of Donaldson [10], Daskalopoulos [9], Råde [39], and AtiyahBott $[\mathbf{1}]$, this is identical to the decomposition by Harder-Narasimhan type of the corresponding holomorphic $G$-bundle. For $K=U(n), \mathcal{A}(P)_{\xi}$ consists of connections such that the corresponding holomorphic bundle has HarderNarasimhan quotients with ranks $r_{j}$ and degrees $d_{j}$.

For each $\xi \in \Xi(P)$, the universal quotient of $\mathcal{A}(P)_{\xi}$ by $G(P)$ is the moduli space $\mathcal{M}\left(X, K_{\xi} ; \xi\right)$ of $K_{\xi}$-bundles with constant central curvature $\xi$. Define a $K$-class on $\nu_{\xi} \rightarrow \mathcal{M}\left(X, K_{\xi} ; s \xi\right)$ by

$$
\left(\nu_{\xi}\right)_{[A]}=\left(H^{1} \ominus H^{0}\right)\left(\frac{\bar{\partial}_{A}, \mathfrak{g}}{\mathfrak{g}_{\xi}}\right) \oplus \frac{\mathfrak{p}_{\xi}}{\mathfrak{g}_{\xi}},
$$

where $\bar{\partial}_{A}$ is the corresponding Dolbeault operator. Except for the factor $\mathfrak{p}_{\xi} / \mathfrak{g}_{\xi}$, this is the virtual normal bundle for the embedding of moduli stacks induced by $G_{\xi} \rightarrow G$; see [44]. The inclusion of $\mathfrak{p}_{\xi} / \mathfrak{g}_{\xi}$ in the definition has to do with the fact that the generic complex automorphism group of a bundle of 
type $\xi$ is the corresponding parabolic $P_{\xi}$, which means that $-\mathfrak{p}_{\xi} / \mathfrak{g}_{\xi}$ appears in the stacky normal bundle $\nu_{\xi}$ but not in the corresponding formula in Section 5 .

Let $K_{\xi}^{\prime}$ denote the identity component of the generic automorphism group for $\mathcal{M}\left(X, K_{\xi} ; \xi\right), K_{\xi}^{\prime \prime}=K_{\xi} / K_{\xi}^{\prime}$. Let $K_{\xi, M}^{\prime \prime}$ denote the (finite) subgroup of $K_{\xi}^{\prime \prime}$ contained in the generic automorphism group. Let $\mathcal{M}\left(X, x, K_{\xi} ; \xi\right)$ denote the moduli space of bundles with framing at a base point $x$. If every point in $\mathcal{M}\left(X, K_{\xi} ; \xi\right)$ has automorphism group $K_{\xi}^{\prime}$, then $M\left(X, x, K_{\xi} ; \xi\right)$ is a locally free $K_{\xi}^{\prime \prime}$-space with quotient $\mathcal{M}\left(X, K_{\xi} ; \xi\right)$. For any $h \in \mathcal{S}\left(\mathfrak{k}_{\xi}\right)^{K_{\xi}}$, let $\kappa_{\xi}(h) \in \mathcal{H}_{K_{\xi}^{\prime}}\left(\mathcal{M}\left(X, K_{\xi} ; \xi\right)\right)$ denote the corresponding characteristic class. Let $\mathcal{L}_{(\xi)}$ denote the $K_{\xi}^{\prime}$-equivariant Liouville form on $\mathcal{M}\left(X, K_{\xi} ; \xi\right)$, with constant moment map with value $\xi$. Let $\mu_{\mathcal{A}(X), \xi} \in \mathcal{S}^{\prime}\left(\mathfrak{k}^{*}\right)^{K}$ denote the distribution defined by

$$
\left(\mu_{\mathcal{A}(X), \xi}, h\right)=\int_{\mathcal{M}\left(X, K_{\xi} ; \xi\right) \times \mathfrak{k}_{\xi}^{\prime}} \mathcal{L}_{(\xi)} \wedge \operatorname{Eul}\left(\nu_{\xi}\right)_{\xi}^{-1} \wedge \kappa_{\xi}\left(\operatorname{Vol}_{K_{\xi}}^{K} \operatorname{Res}_{K_{\xi}}^{K} h\right),
$$

times $\operatorname{Vol}\left(K_{\xi}^{\prime \prime} / K_{\xi, M}^{\prime \prime}\right)$, compare with Theorem 6.1. Let

$$
\Xi(X)=\bigcup_{P} \Xi(P)
$$

and define the Yang-Mills partition function by

$$
Z(X):=\sum_{\xi \in \Xi(X)}\left(\mu_{\mathcal{A}(X), \xi}, h\right),
$$

where $h \in \mathcal{S}\left(\mathfrak{k}^{*}\right)^{K}$ is the Fourier transform of $\hat{h}(\zeta)=\exp \left(-\frac{\epsilon}{2}\|\zeta\|^{2}\right)$. (There is a slight inconsistency with the previous formal definition to the effect of a missing factor of a power of $\epsilon$.)

Some care is needed for the definition in the presence of reducible connections. Let $\mathcal{M}(X, K)_{\nu}$ denote the moduli space of flat $K$ bundles on the once-punctured surface, with holonomy around the puncture conjugate to $\exp (\nu)$. This space admits a holomorphic description in terms of semistable bundles with a parabolic reduction at the puncture, described in MehtaSeshadri [31]. Let $Z(K)$ denote the center of $K$ and $K^{\prime \prime}=K / Z(K)$. The function

$$
Z(X, \nu):=\# \operatorname{Vol}(Z(K)) \operatorname{Vol}\left(\mathcal{M}(X, K)_{\nu}\right)
$$

is piecewise polynomial for $\nu \in \mathfrak{k}^{\prime \prime}$. If every point in $\mathcal{M}(X, K)$ has automorphism group $Z(K)$, then

$$
\mu_{\mathcal{A}(X), 0}=\operatorname{Vol}(K \cdot \nu)^{-1} Z(X, \nu) \mu_{\mathfrak{k}^{\prime \prime}, *}
$$

for $\nu$ in a neighborhood of 0 . In case $\mathcal{M}(X, K)$ contains reducibles, this can be taken as the definition of $\mu_{0}$. There are similar definitions for the other distributions $\mu_{\xi}$ in the presence of reducible connections. 
The main result of this section is

Theorem 9.1. ("Migdal formula", see $[\mathbf{4 6}, 2.51])$ Let $K$ be a compact connected group. The two-dimensional Yang-Mills partition function is given by

$$
Z(X)=\operatorname{Vol}(K)^{2 g} \sum_{\nu}\left(\operatorname{dim} V_{\nu}\right)^{2-2 g} \hat{h}(\nu+\rho),
$$

where the sum is over dominant $\nu$ in the weight lattice $\Lambda^{*}$ plus $\rho$.

Here, $\rho$ is the half-sum of the positive roots which is a weight if $\mathfrak{k}$ is spinnable. Before we give the proof, we note the corollary (as already discussed in $[46])$.

Corollary 9.2. Suppose that $K$ is semisimple and $g \geq 2$. The volume of the moduli space $\mathcal{M}(X, K)$ is

$$
\operatorname{Vol}(\mathcal{M}(X, K))=\# Z(K) \operatorname{dim}(K)^{2 g} \sum_{\nu}\left(\operatorname{dim} V_{\nu}\right)^{2-2 g},
$$

where $Z(K)$ is the center of $K$.

Proof. Take the limit $\epsilon \rightarrow 0$ in Theorem 9.1. By definition of $Z(X)$, the limit

$$
\lim _{\epsilon \rightarrow 0} Z(X)=\# Z(K)^{-1} \operatorname{Vol}(\mathcal{M}(X, K)) .
$$

On the other hand, the limit of the right-hand side of Theorem 9.1 is

$$
\operatorname{dim}(K)^{2 g} \sum_{\nu}\left(\operatorname{dim} V_{\nu}\right)^{2-2 g}
$$

which proves the corollary.

The measures $\mu_{\mathcal{A}(X), \xi}$ for $\xi$ generic can be described as follows. The moduli space $\mathcal{M}\left(X, K_{\xi}, \xi\right)$ is the Jacobian of torus bundles with first Chern class $\xi$ and is diffeomorphic to $T^{2 g}$. The characteristic classes of the bundle $\nu$ are computed in $[\mathbf{4 2}, \mathbf{4 4}$, p. 8]. One obtains

$$
\operatorname{Eul}\left(\nu_{\xi}\right)=(-1)^{2 \rho(\xi)}|\operatorname{Eul}(\mathfrak{k} / \mathfrak{t})|^{2 g-2} .
$$

Integrating over $\mathcal{M}\left(X, K_{\xi}, \xi\right)$ gives

$$
\begin{aligned}
\mu_{\mathcal{A}(X), \xi} & =i^{(2 g-1) \operatorname{dim}(K / T) / 2}(-1)^{2 \rho(\xi)} \operatorname{Ind}_{T}^{K} \int_{T^{2 g}} \exp \left(\omega_{(\xi)}\right) \delta_{\xi} \operatorname{Eul}(\mathfrak{k} / \mathfrak{t})_{\xi}^{1-2 g} \\
& =i^{(2 g-1) \operatorname{dim}(K / T) / 2}(-1)^{2 \rho(\xi)} \operatorname{Ind}_{T}^{K} \operatorname{Vol}\left(T^{2 g}\right) \delta(\xi) \operatorname{Eul}(\mathfrak{k} / \mathfrak{t})_{\xi}^{1-2 g}
\end{aligned}
$$

The proof of Theorem 9.1 is based on the idea, introduced by Teleman [42], that the sum over strata is the same as the sum of contributions from the $T$-bundles. Define

$$
\mu_{\mathcal{A}(X)}:=\sum_{\xi \in \Xi(X)} \mu_{\mathcal{A}(X), \xi} \in \mathcal{D}^{\prime}\left(\mathfrak{k}^{*}\right)^{K}
$$


which is a kind of Duistermaat-Heckman measure for $\mathcal{A}(X)$. Let

$$
\nu_{\mathcal{A}(X), \xi}:=\frac{i^{\left(g-\frac{1}{2}\right) \operatorname{dim}(K / T)}}{\# W_{\xi}}(-1)^{2 \rho(\xi)} \operatorname{Ind}_{T}^{K} \operatorname{Vol}\left(T^{2 g}\right) \delta(\xi) \operatorname{Eul}(\mathfrak{k} / \mathfrak{t})_{\zeta}^{1-2 g}
$$

if $\mathcal{M}\left(X, K_{\xi}, \xi\right)$ contains $T$-bundles, and zero otherwise. Here, $\zeta \in \mathfrak{t}_{+}^{*}$ is any regular element. We wish to compare $\mu_{\mathcal{A}(X)}$ with

$$
\nu_{\mathcal{A}(X)}:=\sum_{\xi \in \Xi(X)} \nu_{\mathcal{A}(X), \xi}
$$

which is the sum of the "fixed-point contributions" from $T$-bundles. For any distribution $\mu \in \mathcal{D}^{\prime}(\mathfrak{k})^{K}$, define a distribution $\mu_{T} \in \mathcal{D}^{\prime}(\mathfrak{t})^{\operatorname{sign}(W)}$ by

$$
\left(\mu_{T}, \operatorname{Vol}_{T}^{K} \operatorname{Res}_{T}^{K} h\right):=(\mu, h) .
$$

The map $\mu \mapsto \mu_{T}$ is a right inverse to $\operatorname{Ind}_{T}^{K}$. We will need the following lemma:

\section{Lemma 9.3.}

a) $\mu_{\mathcal{A}(X), T}$ is invariant under translation by the coweight lattice $\Lambda$ and anti-invariant under $W$. (In other words, anti-invariant under the action of the affine Weyl group.)

b) $\left(\mu_{\mathcal{A}(X), \xi}-\nu_{\mathcal{A}(X), \xi}\right)_{T}$ has Fourier transform supported in $\mathfrak{t}_{\text {sing }}$.

The lemma is obtained by taking the high level limit of the corresponding $K$-theoretic statements in [44]. Since $\mu_{\mathcal{A}(X), T}$ is a periodic distribution, its Fourier transform $\mathcal{F}_{\mathfrak{t}}^{-1} \mu_{\mathcal{A}(X), T}$ is a sum of delta functions at weights:

$$
\mathcal{F}_{\mathfrak{t}}^{-1} \mu_{\mathcal{A}(X), T}=\sum c_{\lambda} \delta_{\lambda}
$$

Since $\mu_{\mathcal{A}(X), T}$ is $W$-anti-invariant, $c_{\lambda}=0$ unless $\lambda$ is regular. By part (b) of Lemma $9.3, \mu_{\mathcal{A}(X), T}$ is equal to $\nu_{\mathcal{A}(X), T}$ plus a distribution whose Fourier transform is supported in $\mathfrak{t}_{\text {sing }}$. We have

$$
\nu_{\mathcal{A}(X), T}=\frac{i^{(g-1 / 2) \operatorname{dim}(K / T)}}{\# W} \sum_{\xi \in \Lambda} \delta_{\xi} \operatorname{Vol}\left(T^{2 g}\right)(-1)^{2 \rho(\xi)} \operatorname{Eul}(\mathfrak{k} / \mathfrak{t})^{1-2 g} .
$$

By the Poisson summation formula

$$
\mathcal{F}_{\mathfrak{t}}^{-1} \mu_{\mathcal{A}(X), T}=i^{-\operatorname{dim}(K / T) / 2} \sum_{\lambda \in \Lambda^{*}+\rho}(\# W)^{-1} \delta_{\lambda} \operatorname{Vol}\left(T^{2 g}\right) \prod_{\alpha>0} 2 \pi(\alpha, \lambda)^{1-2 g},
$$

where (since $c_{\lambda}=0$ for singular $\lambda$ ) the sum is over regular $\lambda$. Hence,

$$
\mu_{\mathcal{A}(X)}=i^{-\operatorname{dim}(K / T) / 2} \operatorname{Vol}(K)^{2 g-1} \operatorname{Vol}(T) \operatorname{Ind}_{T}^{K} \sum_{\lambda} \delta_{\lambda+\rho} \operatorname{dim}\left(V_{\lambda}\right)^{1-2 g},
$$


where the sum is over $\lambda$ such that $\lambda+\rho$ is a dominant weight. (If $\lambda$ is not a weight, $V_{\lambda}$ is a representation of the universal cover of $K$.) Finally, pairing with the Gaussian $h$ gives

$$
\begin{aligned}
\left(\mu_{\mathcal{A}(X)}, h\right) & =\frac{\operatorname{Vol}(K)^{2 g-1} \operatorname{Vol}(T)}{i^{\operatorname{dim}(K / T) / 2}}\left(\sum_{\lambda} \delta_{\lambda+\rho} \operatorname{dim}\left(V_{\lambda}\right)^{1-2 g}, \operatorname{Vol}_{T}^{K} \operatorname{Res}_{T}^{K} \hat{h}\right) \\
& =\left(\operatorname{Vol}(K)^{2 g} \sum_{\lambda} \delta_{\lambda+\rho} \operatorname{dim}\left(V_{\lambda}\right)^{2-2 g}, \operatorname{Res}_{T}^{K} \hat{h}\right) \\
& =\operatorname{Vol}(K)^{2 g} \sum_{\lambda} \operatorname{dim}\left(V_{\lambda}\right)^{2-2 g} \hat{h}(\lambda+\rho)
\end{aligned}
$$

which completes the proof of 9.1. This computation is done on a physics level of rigor by Blau-Thompson [7]. The main point is that the contribution of the semistable stratum is not affine Weyl-invariant, but only becomes so after adding the contributions from the higher strata. As in [44], the additional symmetry removes the necessity of doing any hard computations, that is, any integrals other than integrals over Jacobians.

Example 9.4. Let $K=S U(2)$ and identify $\mathfrak{t} \rightarrow \mathbb{R}$ so that the weight lattice is $\mathbb{Z} / 2$ and coweight lattice $\mathbb{Z}$. If $X$ has genus $g=1$, an explicit computation shows

$$
Z(X, \nu)=\frac{1}{4} \operatorname{Vol}\left(T^{2}\right)(1-2 \nu)
$$

For $\xi$ a positive integer, $K_{\xi}=K$ and $\mathcal{M}(X, T ; \xi)=T^{2}$. The normal bundle $\nu_{\xi}=(\mathfrak{k} / \mathfrak{t})^{2}$, hence

$$
\begin{aligned}
\mu_{\mathcal{A}(X), \xi} & =\frac{1}{2} \operatorname{Ind}_{T}^{K} \operatorname{Vol}\left(T^{2}\right) \delta(\xi) \operatorname{Eul}(\mathfrak{k} / \mathfrak{t})_{+}^{-1}-\delta(-\xi) \operatorname{Eul}(\mathfrak{k} / \mathfrak{t})_{-}^{-1} \\
& =\frac{1}{2} \operatorname{Vol}\left(T^{2}\right)\left(\delta(\xi) H_{+}-\delta(-x) H_{-}\right)
\end{aligned}
$$

where $H_{ \pm}$are the Heaviside distributions. The sum is the sawtooth distribution shown below in solid lines in Figure 5. The dotted line is the contribution from $\xi=0$.

It seems an interesting question whether a similar definition could be used for other path integrals, for instance, holomorphic Yang-Mills theory in four dimensions. On the other hand, other path integrals such as full fourdimensional Yang-Mills or two-dimensional Yang-Mills with observables do not seem to admit heuristic interpretations as pairings in equivariant cohomology, and it appears unlikely that the techniques described here would apply. 


\section{Appendix A. Convergence of the gradient flow}

This section contains a proof that the norm-square of minus the gradient flow of the moment map converges. This result appeared some time ago in an unpublished manuscript by Duistermaat, who used the gradient inequality of Lojasiewicz [28]; see Lerman [25]. Here, we prove the necessary gradient inequality directly, using the local model (3.18), and obtain explicit estimates for the rate of convergence. The same estimates were established for the infinite dimensional Yang-Mills heat flow by Råde [39]; our goal is to put the finite dimensional case on equal footing.

First, we discuss some background on gradient flows. Let $M$ be a Riemannian manifold with metric $g, f \in C^{\infty}(M)$ and $\operatorname{grad}(f)$ its gradient. Let crit $(f)$ the critical set of $f$. For any $x \in \operatorname{crit}(f)$, let $L(f, x)$ be the set of $\gamma>0$ such that there exists a neighborhood $U$ of $x$ and a constant $C>0$ so that for all $m \in U$,

$$
\|\operatorname{grad}(f)(m)\|>C|f(m)-f(x)|^{\gamma} .
$$

Theorem A.1 (Lojasiewicz gradient inequality). If $f: \mathbb{R}^{n} \rightarrow \mathbb{R}$ is a realanalytic function, then for every critical point $x$ of $f$ there exists a $\gamma \in$ $L(f, x)$ with $\gamma \in\left[\frac{1}{2}, 1\right)$.

The Riemannian metric on $\mathbb{R}^{n}$ is assumed to be the standard one. However, the same inequality holds for an arbitrary metric, with the same exponent but possibly different constant. Therefore, the same result holds for functions on Riemannian manifolds that are real-analytic near their critical sets. We will not use Theorem A.1 in this paper but instead a special case which is easy to prove:

Lemma A.2. Let $f: \mathbb{R}^{n} \rightarrow \mathbb{R}$ be a homogeneous polynomial of degree $d$, $1-1 / d \in L(f, 0)$.

Proof. Let $v \in \mathbb{R}^{n}$ have norm 1 and $f_{v}(t)=f(t v)$. Then

$$
\|\operatorname{grad}(f)(t v)\| \geq\left|f_{v}^{\prime}(t)\right|=(d-1)\left|f_{v}(t)\right|^{1-1 / d}=(d-1)|f(t v)|^{1-1 / d} .
$$

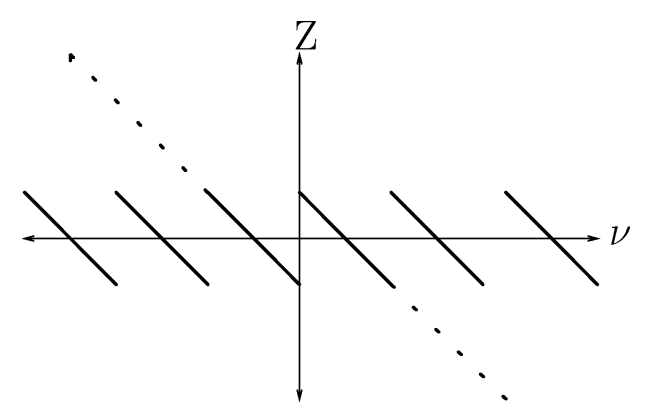

Figure 5. One-point function for genus one, $K=S U(2)$. 
The following theorems are probably well known. Suppose that $f$ is proper and bounded from below. Since $f^{-1}(-\infty, c]$ is compact for any $c>0$, the flow $\varphi_{t}: M \rightarrow M$ of $-\operatorname{grad}(f)$ is defined for all times $t$.

Theorem A.3. Let $c$ be a critical value of $f$. Suppose that there exists $\gamma \in(0,1)$ such that $\gamma \in L(f, x)$ for every $x \in \operatorname{crit}(f) \cap f^{-1}(c)$.

a) Any trajectory $m_{t}$ of $-\operatorname{grad}(f)$ such that $f\left(m_{t}\right) \rightarrow c$ has a unique limit $m_{\infty}$ as $t \rightarrow \infty$.

b) Let $W_{c}$ denote the stable set of points $m \in M$ with $m_{\infty} \in f^{-1}(c)$. The map $m \rightarrow m_{\infty}$ is a deformation retraction of $W_{c}$ onto $f^{-1}(c) \cap \operatorname{crit}(f)$.

Theorem A.4. For any $m \in M$ and $\gamma \in L\left(f, m_{\infty}\right)$, there exist constants $C$ and $k$ and a time $T$ such that if $t>T$, then

a) if $\gamma \in\left(\frac{1}{2}, 1\right)$ then $d\left(m_{t}, m_{\infty}\right) \leq C t^{(\gamma-1) /(2 \gamma-1)}$, and

b) if $\gamma=\frac{1}{2}$ then $d\left(m_{t}, m_{\infty}\right) \leq C e^{-k t}$.

The following is a sketch of proof, see also [25]. Since $f$ is proper, there exists a neighborhood $U_{c}$ of $f^{-1}(c) \cap \operatorname{crit}(f)$ such that if $m \in U_{c}$, then

$$
\|\operatorname{grad}(f)(m)\| \geq(f(m)-c)^{\gamma} .
$$

Let $m \in M$ and $m_{t}$ the trajectory of $-\operatorname{grad}(f)$ with $m(0)=m$. Using properness of $f$ again, there exists $T>0$ such that $m_{t}$ lies in some $U_{c}$ for $t>T$. For $t>T$,

$$
\begin{aligned}
\frac{d}{d t}\left(f\left(m_{t}\right)-c\right)^{1-\gamma} & =-(1-\gamma)\left\|\operatorname{grad}(f)\left(m_{t}\right)\right\|^{2}\left(f\left(m_{t}\right)-c\right)^{-\gamma} \\
& \geq-C\left\|\operatorname{grad}(f)\left(m_{t}\right)\right\|
\end{aligned}
$$

by (A.1). Hence, for $t_{1}, t_{2}>T$,

$$
\begin{aligned}
d\left(m\left(t_{1}\right), m\left(t_{2}\right)\right) & \leq \int_{t_{1}}^{t_{2}} d t \| \operatorname{grad}\left(f\left(m_{t}\right) \|\right. \\
& \leq C\left(\left(f\left(m\left(t_{1}\right)\right)-c\right)^{1-\gamma}-\left(f\left(m\left(t_{2}\right)\right)-c\right)^{1-\gamma}\right) .
\end{aligned}
$$

By the Cauchy criterion, $m_{t}$ converges to a critical point $m_{\infty} \in f^{-1}(c)$. (A.2) and (A.3) also imply that for $t>T$

$$
d\left(m_{t}, m_{\infty}\right) \leq C\left(f\left(m_{t}\right)-c\right)^{1-\gamma} .
$$

Next, we show that the map $W_{c} \rightarrow f^{-1}(c) \cap \operatorname{crit}(f), m \mapsto m_{\infty}$ is a deformation retraction, that is, that $W_{c} \times[0, \infty] \rightarrow W_{c}, m \mapsto m_{t}$ is continuous. Let $\epsilon>0$. By (A.4), there exists $\delta>0$ so that $f(m)-c<\delta$ and $m \in W_{c}$ imply $d\left(m, m_{\infty}\right)<\epsilon / 3$. Fix $m_{1} \in W_{c}$ and let $t$ be sufficiently large so that $f\left(m_{1, t}\right)-c<\delta$. By smooth dependence on initial conditions, if $m_{2}$ is sufficiently close to $m_{1}$, then $d\left(m_{1, t}, m_{2, t}\right)<\epsilon / 3$. For $\epsilon$ sufficiently small, 
$f\left(m_{2, t}\right)-c<\delta$. Hence, if $m_{2}$ lies in $W_{c}$ and is sufficiently close to $m_{1}$, then

$$
\begin{aligned}
d\left(m_{1, \infty}, m_{2, \infty}\right) & \leq d\left(m_{1, \infty}, m_{1, t}\right)+d\left(m_{1, t}, m_{2, t}\right)+d\left(m_{2, t}, m_{2, \infty}\right) \\
& <\frac{\epsilon}{3}+\frac{\epsilon}{3}+\frac{\epsilon}{3}=\epsilon
\end{aligned}
$$

and also $d\left(m_{2, t}, m_{1, \infty}\right)<\epsilon$. This completes the proof of Theorem A.3.

Going back to (A.1), we have

$$
\frac{d}{d t}\left(f\left(m_{t}\right)-c\right)=-\|\operatorname{grad}(f)(m)\|^{2} \leq-C\left(f\left(m_{t}\right)-c\right)^{2 \gamma} .
$$

Integrating gives

$$
f\left(m_{t}\right)-c \leq\left\{\begin{array}{ll}
C(t-T)^{-1 /(2 \gamma-1)} & \gamma \in\left(\frac{1}{2}, 1\right) \\
C e^{-k(t-T)} & \gamma=\frac{1}{2}
\end{array}\right\} .
$$

Using (A.4), this proves Theorem A.4.

We apply Theorems A.3 and A.4 to the norm-square of the moment map. Let $K$ be a compact Lie group with Lie algebra $\mathfrak{k}$, and $():, \mathfrak{k} \times \mathfrak{k} \rightarrow \mathbb{R}$ an invariant inner product. From now on, we use the inner product to identify $\mathfrak{k}$ with its dual $\mathfrak{k}^{*}$. Let $M$ be a Hamiltonian $K$-manifold with proper moment $\operatorname{map} \Phi: M \rightarrow \mathfrak{k}$ and $f=\frac{1}{2}(\Phi, \Phi)$. Choose an invariant compatible almost complex structure $J$, so that $\omega(\cdot, J \cdot)$ defines a $K$-invariant Riemannian metric $g$ on $M$. For each $\xi \in \Xi(M)$, let $C_{\xi}$ denote the corresponding component (not necessarily connected) of $\operatorname{crit}(f)$ and $Z_{\xi}$ the union of components of the fixed point set of $U(1)_{\xi}$ meeting $\Phi^{-1}(\xi)$.

Lemma A.5. Let $x$ be a point in $\Phi^{-1}(\xi) \cap \operatorname{crit}(f)$. (a) $\frac{3}{4} \in L(f, x)$. (b) If $x$ lies in the principal orbit-type stratum of $Z_{\xi}$, then $\frac{1}{2} \in L(f, x)$.

We apply the local model (3.18) to $y=x \in \operatorname{crit}(f)$, so that $\xi \in \mathfrak{k}_{x}$. We identify a neighborhood of $x$ in $M$ with a neighborhood of 0 in $\mathfrak{k}_{x}^{\perp} \oplus T_{0} S \oplus$ $\left(\mathfrak{k}_{\xi}^{*} \cap \mathfrak{k}_{x}^{\circ}\right)$. We denote by $g_{0}$ the constant metric that is the direct sum of the metric on $T_{0} S$ and the metrics on $\mathfrak{k}_{x}^{\perp} \oplus\left(\mathfrak{k}_{\xi}^{*} \cap \mathfrak{k}_{x}^{\circ}\right)$ induced by the metric on $\mathfrak{k}$. Let $\|\cdot\|_{0}$ denote the corresponding norm; we will denote by the same notation its restriction to $T_{0} S$. Let $m \in M$ and $[k, s, \nu]$ be the image of $m$ in $U^{\prime}$. Since $f$ is $K$-invariant, it suffices to consider the case $k=1$. For $m$ sufficiently close to $x$, we have

$$
\begin{aligned}
\|\operatorname{grad}(f)(m)\| & =\left\|\Phi(m)_{M}(m)\right\| \\
& \geq C_{1}\left\|\Phi(m)_{M}(m)\right\|_{0} \\
& =C_{1}\left\|\left(\nu,\left(\Phi_{S}(s)+\xi\right) \cdot s,\left(\Phi_{S}(s)+\xi\right) \cdot \nu\right)\right\|_{0} \\
& =C_{1}\left(\left\|\left(\xi+\Phi_{S}(s)\right) \cdot s\right\|_{0}^{2}+\|\nu\|^{2}\right)^{1 / 2} .
\end{aligned}
$$

Let $S_{0} \subset S$ denote the fixed point set of $K_{x}, S_{1}$ the symplectic complement of $S_{0}$ in the fixed point set of $K_{\xi}$, and $S_{2}$ the symplectic complement of 
$S_{1} \oplus S_{2}$ so that $S=S_{0} \oplus S_{1} \oplus S_{2}$. Since $\Phi$ vanishes on $S_{0}, f$ and $\operatorname{grad}(f)$ are independent of $s_{0} \in S_{0}$. $f$ is homogeneous of degree 4 on $S_{1}$. By Lemma A.2

$$
\begin{aligned}
\left\|\Phi_{S}\left(s_{0}, s_{1}, 0\right) \cdot\left(s_{0}, s_{1}, 0\right)\right\|_{0}^{2} & =\left\|\operatorname{grad}\left(\Phi_{S}, \Phi_{S}\right)\left(s_{0}, s_{1}, 0\right) / 2\right\|_{0}^{2} \\
& \geq C_{2}\left\|\Phi_{S}\left(s_{0}, s_{1}, 0\right)\right\|^{3} .
\end{aligned}
$$

We expand

$$
\begin{aligned}
\left\|\left(\xi+\Phi_{S}(s)\right) \cdot s\right\|_{0}^{2}= & \left\|\Phi_{S}\left(s_{0}, s_{1}, 0\right) \cdot\left(s_{0}, s_{1}, 0\right)\right\|_{0}^{2}+2 g_{0}\left(\Phi_{S}\left(0,0, s_{2}\right)\right. \\
& \left.\cdot\left(s_{0}, s_{1}, 0\right), \Phi_{S}\left(s_{0}, s_{1}, 0\right) \cdot\left(s_{0}, s_{1}, 0\right)\right) \\
& +\left\|\left(\xi+\Phi_{S}\left(s_{0}, s_{1}, 0\right)\right) \cdot s_{2}\right\|_{0}^{2} \\
& +\left\|\Phi_{S}\left(0,0, s_{2}\right) \cdot\left(s_{0}, s_{1}, 0\right)\right\|_{0}^{2}+2 g_{0}\left(\left(\xi+\Phi_{S}\left(s_{0}, s_{1}, 0\right)\right)\right. \\
& \left.\cdot\left(0,0, s_{2}\right), \Phi_{S}\left(0,0, s_{2}\right) \cdot\left(0,0, s_{2}\right)\right) \\
& +\left\|\Phi_{S}\left(0,0, s_{2}\right) \cdot\left(0,0, s_{2}\right)\right\|_{0}^{2}
\end{aligned}
$$

which are terms of degree $0,2,2,4,4,6$, respectively, in $s_{2}$. Because $\xi$ acts on $S_{2}$ without fixed points, the terms of degree 2 sum to a positive quadratic form for $s_{1}$ sufficiently small. It follows that

$$
\left\|\left(\xi+\Phi_{S}(s)\right) \cdot s\right\|_{0}^{2} \geq C_{3}\left(\left\|\Phi_{S}\left(s_{0}, s_{1}, 0\right)\right\|^{3}+\left\|s_{2}\right\|_{0}^{2}\right)
$$

for $s_{1}, s_{2}$ sufficiently small. By equation (3.19)

$$
\begin{aligned}
f(m)-f(x)= & \frac{1}{2}\left\|\Phi_{S}(s)+\xi\right\|^{2}+\frac{1}{2}\|\nu\|^{2}-\frac{1}{2}\|\xi\|^{2} \\
= & \frac{1}{2}\left\|\Phi_{S}(s)\right\|^{2}+\left(\Phi_{S}(s), \xi\right)+\frac{1}{2}\|\nu\|^{2} \\
= & \frac{1}{2}\left\|\Phi_{S}\left(s_{0}, s_{1}, 0\right)\right\|^{2}+\left(\Phi_{S}\left(s_{0}, s_{1}, 0\right), \Phi_{S}\left(0,0, s_{2}\right)\right) \\
& +\frac{1}{2}\left\|\Phi_{S}\left(0,0, s_{2}\right)\right\|^{2}+\left(\xi, \Phi_{S}\left(0,0, s_{2}\right)\right)+\frac{1}{2}\|\nu\|^{2}
\end{aligned}
$$

which is bounded by $\frac{1}{2}\left\|\Phi_{S}\left(s_{0}, s_{1}, 0\right)\right\|^{2}+C_{4}\left\|s_{2}\right\|^{2}+\frac{1}{2}\|\nu\|^{2}$. For $a, b \in[0,1]$, we have $\left(a^{3}+b^{2}\right)^{2} \geq\left(a^{2}+b^{2}\right)^{3} / 2$. Applying this inequality with $a=$ $\left\|\Phi_{S}\left(s_{0}, s_{1}, 0\right)\right\|, b=\left(\left\|s_{2}\right\|_{0}^{2}+\|\nu\|^{2}\right)^{1 / 2}$ gives

$$
\begin{aligned}
\|\operatorname{grad}(f)\| & \geq C_{5}\left(\left\|\Phi_{S}\left(s_{0}, s_{1}, 0\right)\right\|^{3}+\left\|s_{2}\right\|_{0}^{2}+\|\nu\|^{2}\right)^{1 / 2} \\
& \geq C_{6}\left(\left\|\Phi_{S}\left(s_{0}, s_{1}, 0\right)\right\|^{2}+\left\|s_{2}\right\|_{0}^{2}+\|\nu\|^{2}\right)^{3 / 4} \\
& \geq C_{7}(f(m)-f(x))^{3 / 4}
\end{aligned}
$$

which completes the proof of part (a) of the lemma. To prove (b), note that if $x$ is contained in the principal orbit-type stratum of $Z_{\xi}$, then $S_{1}$ is trivial. Hence for $t>T$,

$$
\|\operatorname{grad}(f)\| \geq C\left(\left\|s_{2}\right\|_{0}^{2}+\|\nu\|^{2}\right)^{1 / 2} \geq C\left(f\left(m_{t}\right)-c\right)^{1 / 2}
$$

It now follows from Theorem A.3 


\section{Theorem A.6.}

a) Every trajectory $m_{t}$ of $-\operatorname{grad}(f)$ converges to a point $m_{\infty} \in \operatorname{crit}(f)$.

b) For all $\xi \in \Xi(M)$, the map $m \mapsto m_{\infty}$ is a deformation retraction of $M_{\xi}$ onto $C_{\xi}$.

c) If $m_{\infty}$ is contained in the principal orbit-type stratum of $Z_{\xi}$ then there exist constants $C, k, T>0$ such that $d\left(m_{t}, m_{\infty}\right)<C e^{-k t}$ for $t>T$. Otherwise, there exist constants $C, T>0$ such that $d\left(m_{t}, m_{\infty}\right)<C t^{-\frac{1}{2}}$ for $t>T$.

\section{References}

[1] M.F. Atiyah and R. Bott, The Yang-Mills equations over Riemann surfaces, Phil. Trans. Roy. Soc. London Ser. A 308 (1982), 523-615.

[2] M.F. Atiyah and R. Bott, The moment map and equivariant cohomology, Topology 23(1) (1984), 1-28.

[3] S. Axelrod and I.M. Singer, Chern-Simons perturbation theory. II. J. Differential Geom., 39(1) (1994), 173-213.

[4] N. Berline, E. Getzler, and M. Vergne, Heat kernels and dirac operators, volume 298 of Grundlehren der mathematischen Wissenschaften, Springer-Verlag, BerlinHeidelberg-New York, 1992.

[5] N. Berline and M. Vergne, Zéro d'un champ de vecteurs et classes caractéristiques équivariantes, Duke Math. J. 50 (1983), 539-549.

[6] A. Białynicki-Birula, Some theorems on actions of algebraic groups, Ann. Math. (2) 98 (1873), 480-497.

[7] M. Blau and G. Thompson, Localization and diagonalization: a review of functional integral techniques for low-dimensional gauge theories and topological field theories, in Functional integration (Cargèse, 1996), Vol. 361 of NATO Adv. Sci. Inst. Ser. B Phys., pages 363-410, Plenum, New York, 1997.

[8] A. Canas da Silva and V. Guillemin, On the Kostant multiplicity formula for group actions with non-isolated fixed points, Adv. Math. 123(1) (1996) 1-15.

[9] G.D. Daskalopoulos, The topology of the space of stable bundles on a compact Riemann surface, J. Differential Geom. 36(3) (1992) 699-746.

[10] S.K. Donaldson, A new proof of a theorem of Narasimhan and Seshadri, J. Differential Geom. 18(2) (1983) 269-277.

[11] J.J. Duistermaat, Equivariant cohomology and stationary phase, in Symplectic geometry and quantization, (Sanda and Yokohama, 1993) Vol. 179 of Contemp. Math., pages 45-62, Providence, RI, Amer. Math. Soc., 1994.

[12] D.S. Freed and R.E. Gompf, Computer calculation of Witten's 3-manifold invariant, Comm. Math. Phys. 141(1) (1991), 79-117.

[13] V. Guillemin, E. Lerman and S. Sternberg, On the Kostant multiplicity formula, J. Geom. Phys. 5(4) (1988) 721-750.

[14] V. Guillemin, E. Lerman and S. Sternberg, Symplectic fibrations and multiplicity diagrams, Cambridge University Press, Cambridge, in press.

[15] Victor Guillemin, Eugene Lerman, and Shlomo Sternberg, Symplectic fibrations and multiplicity diagrams, Cambridge University Press, Cambridge, 1996. 
[16] V.W. Guillemin and S. Sternberg, Supersymmetry and equivariant de Rham theory, Springer-Verlag, Berlin, 1999. With an appendix containing two reprints by Henri Cartan [MR 13,107e; MR 13,107f].

[17] R. Hartshorne, Residues and duality. Lecture notes of a seminar on the work of A. Grothendieck, given at Harvard 1963/64. With an appendix by P. Deligne. Lecture Notes in Mathematics, No. 20, Springer-Verlag, Berlin, 1966.

[18] P. Heinzner and F. Loose, Reduction of complex Hamiltonian G-spaces, Geom. Funct. Anal. 4(3) (1994), 288-297.

[19] L. Hörmander. The analysis of linear partial differential operators. II. Springer-Verlag, Berlin, 1983. Differential operators with constant coefficients.

[20] L.C. Jeffrey and F.C. Kirwan, Localization for nonabelian group actions, Topology 34 (1995) 291-327.

[21] L.C. Jeffrey and F.C. Kirwan, Intersection theory on moduli spaces of holomorphic bundles of arbitrary rank on a Riemann surface, Ann. Math. (2), 148(1) (1998) 109-196.

[22] F.C. Kirwan, Cohomology of quotients in symplectic and algebraic geometry, Vol. 31 of Mathematical Notes, Princeton Univ. Press, Princeton, 1984.

[23] F.C. Kirwan, Convexity properties of the moment mapping, III, Invent. Math. 77 (1984) 547-552.

[24] S. Kumar and M. Vergne, Equivariant cohomology with generalized coefficients, Astérisque 215 (1993) 109-204.

[25] Eugene Lerman, Gradient flow of the norm squared of a moment map. Enseign. Math. (2), 51(1-2) (2005), 117-127.

[26] E. Lerman, E. Meinrenken, S. Tolman and C. Woodward, Non-abelian convexity by symplectic cuts. Topology, 37 (1998) 245-259.

[27] T. Lévy, Yang-Mills measure on compact surfaces, Mem. Am. Math. Soc. 166(790) (2003), xiv+122.

[28] S. Lojasiewicz, Sur les ensembles semi-analytiques, in 'Actes du Congrès International des Mathématiciens (Nice, 1970)', Tome 2, pages 237-241, Gauthier-Villars, Paris, 1971.

[29] C.-M. Marle, Le voisinage d'une orbite d'une action hamiltonienne d'un groupe de Lie, in South Rhone seminar on geometry, II (Lyon, 1983); Travaux en Cours, pages 19-35, Hermann, Paris, 1984.

[30] S. Martin, Symplectic quotients by a nonabelian group and by its maximal torus, math.SG/0001002.

[31] V.B. Mehta and C.S. Seshadri, Moduli of vector bundles on curves with parabolic structure, Math. Ann. 248 (1980) 205-239.

[32] E. Meinrenken, Witten's formulas for intersection pairings on moduli spaces of flat G-bundles, Adv. Math., 197(1) (2005), 140-197.

[33] E. Meinrenken, Symplectic surgery and the Spin ${ }^{\mathrm{c}}$-Dirac operator, Adv. Math. 134 (1998), 240-277.

[34] L. Ness, A stratification of the null cone via the moment map, Am. J. Math. 106(6) (1984) 1281-1329, with an appendix by D. Mumford.

[35] P.-E. Paradan, Formules de localisation en cohomologie equivariante, Compositio Math. 117(3) (1999) 243-293. 
[36] P.-E. Paradan, The moment map and equivariant cohomology with generalized coefficients, Topology 39(2) (2000), 401-444.

[37] P.-E. Paradan, Localization of the Riemann-Roch character, J. Funct. Anal. 187(2) (2001) 442-509.

[38] E. Prato and S. Wu, Duistermaat-Heckman measures in a non-compact setting, Compositio Math. 94(2) (1994) 113-128.

[39] J. Råde, On the Yang-Mills heat equation in two and three dimensions, J. Reine Angew. Math. 431 (1992), 123-163.

[40] N. Reshetikhin and V.G. Turaev, Invariants of 3-manifolds via link polynomials and quantum groups, Invent. Math. 103(3) (1991), 547-597.

[41] M. Shub, A. Fathi and R. Langevin, Global stability of dynamical systems, SpringerVerlag, New York, 1987. Translated from the French by Joseph Christy.

[42] Constantin Teleman, K-theory and the moduli space of bundles on a surface and deformations of the Verlinde algebra. In Topology, geometry and quantum field theory, volume 308 of London Math. Soc. Lecture Note Ser., pages 358-378. Cambridge Univ. Press, Cambridge, 2004.

[43] C. Teleman, The quantization conjecture revisited, Ann. Math. (2) 152(1) (2000), $1-43$.

[44] C. Teleman and C.T. Woodward, The index formula on the moduli of G-bundles, math.AG/0312154.

[45] M. Vergne, Multiplicities formula for geometric quantization I, II, Duke Math. J. 82 (1996), 143-194,.

[46] E. Witten, On quantum gauge theories in two dimensions, Comm. Math. Phys. 141 (1991), 153-209.

[47] E. Witten, Two-dimensional gauge theories revisited, J. Geom. Phys. 9 (1992), 303-368.

Mathematics-Hill Center

Rutgers University

110 Frelinghuysen RoAd

PiscataWAY, NJ 08854-8019

E-mail address: ctw@math.rutgers.edu

Thanks to the Mathematics Department of the University of Otago, Dunedin, New Zealand for its hospitality while writing this paper, to P.-E. Paradan for explaining his work to me and pointing out a number of mistakes in an earlier version, and to M. Harada, E. Meinrenken, and C. Teleman for helpful discussions. This work was partially supported by NSF grant DMS0093647. 